\title{
DEFORMATION OF COMPLEX STRUCTURE ON NORMAL ISOLATED SINGULARITIES
}

\author{
Kimio MIYAJIMA
}

(Received 12 August 2005)

\begin{abstract}
We give an analytic construction of the versal deformation of normal isolated singularities of dimension greater than or equal to three, by means of deformation of complex structure on its regular part.
\end{abstract}

\section{Introduction}

Complex structure on the regular part is one of main topics of normal singularities. However, the construction of the versal deformation of normal isolated singularities in that approach is still unsolved except for the partial answer in [Ak1] and [Ak2] under the assumption that the dimension $\geq 4$ and the depth $\geq 3$ (cf. [Mi1, Mi2]). In this paper, we show that 'the stably embeddable deformation of complex structure' enables us to clear the main difficulty that required those assumptions. The main theorem of this paper is as follows: let $V$ be a normal analytic sub-variety of a ball $B(c):=\left\{\left.w \in \mathbb{C}^{N}\left|\sum_{\beta=1}^{N}\right| w^{\beta}\right|^{2}<c\right\}$ with only isolated singularity at the origin $o$. Denote by $U:=V \backslash o$ the regular part of $V$. We fix a $C^{\infty}$-framing $\bar{\Omega}:=\left\{\left.w \in U\left|a \leq \sum_{\beta=1}^{N}\right| w^{\beta}\right|^{2} \leq b\right\}$ for some $0<a<b<c$ such that $d\left(\sum_{\beta=1}^{N}\left|w^{\beta}\right|^{2}\right) \neq 0$ on $\bar{\Omega}$ and consider deformation of the complex structure on $\bar{\Omega}$ which are stably embeddable in $\mathbb{C}^{N}$.

MAIN TheOREM. Let $(V, o)$ be a germ of normal Stein space with $\operatorname{dim}_{\mathbb{C}} V \geq 3$ and $\bar{\Omega}$ be as above. Then, there exists a Kuranishi semi-universal family of stably embeddable deformations of the complex structure on $\bar{\Omega}$, which is an open part of the semi-universal family of flat deformations of $V$.

2000 Mathematics Subject Classification: Primary 32G05, 32S30.

Keywords and Phrases: deformation; complex structure; isolated singularity.

This article is an invited contribution to a special issue of the Kyushu Journal of Mathematics commemorating the sixtieth volume. 
The essential idea proving the main theorem is the same as the Cauchy-Riemann (CR)-case. In particular, the part of the formal construction of the semi-universal family is essentially parallel to [Mi3]. However, the argument in the convergence procedure requires a new technique and the main purpose of this paper is to show how to clear the difficulty in that procedure.

We explain the main analytical difficulty and the idea of this paper in overcoming it. For the simplicity of the argument in the following explanation, we will assume that no obstruction appears in our algorithm constructing the Kuranishi semi-universal family. The typical model of our argument is the classical Kodaira-Spencer's method for constructing the semi-universal family of deformations of a compact complex manifold. Let $X$ be a compact complex manifold. Then, the Kodaira-Spencer's method provides a way to construct a family of solutions of $\bar{\partial} \phi-\frac{1}{2}[\phi, \phi]=0$, $\phi \in A_{X}^{0,1}\left(T^{1,0} X\right)$, such that $\phi(t)=\sum_{\alpha=1}^{d} \phi_{\alpha} t_{\alpha} \bmod \mathfrak{m}^{2}$, where $\left\{\phi_{\alpha}\right\}_{\alpha=1, \ldots, d}$ form a cohomology basis of $H^{1}\left(X, T^{1,0} X\right)$ and $\mathfrak{m}$ denotes the maximal ideal of $\mathbb{C}\left\{t_{1}, \ldots, t_{d}\right\}$.

After determining a polynomial $\phi^{(\mu-1)}(t) \in A_{X}^{0,1}\left(T^{1,0} X\right)\left[t_{1}, \ldots, t_{d}\right]$ of degree $\mu-1$ satisfying $\bar{\partial} \phi^{(\mu-1)}(t)-\frac{1}{2}\left[\phi^{(\mu-1)}(t), \phi^{(\mu-1)}(t)\right] \equiv 0 \bmod \mathfrak{m}^{\mu}$, we solve the equation

$$
\bar{\partial} \phi_{\mu}(t)=p_{\mu}(t)
$$

where $p_{\mu}(t)$ is the $\mu$ th homogeneous term of $\frac{1}{2}\left[\phi^{(\mu-1)}(t), \phi^{(\mu-1)}(t)\right]$.

Under the assumption $H^{2}\left(X, T^{1,0} X\right)=0$, we can solve it by

$$
\phi_{\mu}(t)=\bar{\partial}^{*} G p_{\mu}(t)
$$

with $G$ denoting the Green's operator. Then, the convergence of $\lim _{\mu \rightarrow \infty} \phi^{(\mu)}(t)$ is shown by the argument

$$
\left\|\phi_{\mu}\right\|_{k}(t)=\left\|\bar{\partial}^{*} G p_{\mu}\right\|_{k}(t) \ll C\left\|p_{\mu}\right\|_{k-1}(t) \ll C^{\prime}\left\|\phi^{(\mu-1)}\right\|_{k}(t)^{2}
$$

where $\|\cdot\|_{k}$ denotes the Sobolev $k$-norm and refer to Section 3 for the notation $\|\phi\|(t)$ and the symbol $\ll$. It follows from the following estimates:

$$
\|G p\|_{k} \leq C\|p\|_{k-2} \quad \text { and } \quad\|[\phi, \phi]\|_{k-1} \leq C\|\phi\|_{k-1}\|\phi\|_{k} .
$$

In the case of deformation of complex structure on an open domain $\Omega$, this estimate fails for the Neumann operator $N$. The difficulty arises in the estimate of $N p_{\mu}(t)$ near the boundary $\partial \Omega$. In the case of a strongly pseudo-convex domain $\Omega$ with $\operatorname{dim}_{\mathbb{C}} \Omega \geq 4$, Akahori [Ak1] cleared this difficulty using a new norm $\|\phi\|_{(0, k)}^{\prime}$, which is essentially equal to $\|\bar{\partial} \phi\|_{(0, k)}+\left\|\bar{\partial}^{*} \phi\right\|_{(0, k)}+\|\phi\|_{(0, k)}$ near $\partial \Omega$ and to $\|\phi\|_{k+1}$ on 
the interior relatively compact domain where $\|\phi\|_{(0, k)}$ denotes the tangential Sobolev norm, and obtained the estimates

$$
\begin{aligned}
\left\|\bar{\partial}^{*} N p_{\mu}\right\|_{(0, k)}^{\prime}(t) & \ll C\left\|p_{\mu}\right\|_{(0, k)}(t), \\
\left\|\left[\phi_{\mu}, \phi_{\mu}\right]\right\|_{(0, k)}(t) & \ll\left\|\phi^{(\mu-1)}\right\|_{\left(\frac{1}{2}, k\right)}(t)\left\|\phi^{(\mu-1)}\right\|_{(0, k)}^{\prime}(t)
\end{aligned}
$$

if $\phi^{(\mu-1)}(t)_{\mid \partial \Omega}$ is ${ }^{\circ} \tilde{T}^{\prime} U$-valued (cf. Section 3 for the bundle ${ }^{\circ} \tilde{T}^{\prime} U$ ). We remark that the loss of the differentiability in the second inequality causes no trouble since it is taken in by the estimate $\|\phi\|_{\left(\frac{1}{2}, k\right)} \leq C\|\phi\|_{(0, k)}^{\prime}$.

The last trick does not work in our deformation theory: the stably embeddable deformation of complex structure. In our deformation theory, we consider deformation of the pair of a complex structure and an embedding into $\mathbb{C}^{N}$ which is holomorphic with respect to that complex structure. A perturbation of the reference pair is represented by a pair $(\phi, f) \in A_{\bar{\Omega}}^{0,1}\left(T^{1,0} U\right) \oplus A_{\bar{\Omega}}^{0}\left(T^{1,0} \mathbb{C}_{\mid U}^{N}\right)$ satisfying $(\bar{\partial} \phi-$ $\left.\frac{1}{2}[\phi, \phi],(\bar{\partial}-\phi)\left(f_{0}+f\right)\right)=(0,0)$ where $f_{0} \in \bigoplus^{N} H^{0}\left(\bar{\Omega}, \mathcal{O}_{U}\right)$ represents a reference embedding $f_{0}: V \hookrightarrow \mathbb{C}^{N}$. As in the case of stably embeddable deformation of CR structure, the essential part of the above two equations is $(\bar{\partial}-\phi)\left(f_{0}+f\right)=0$. Hence, according to the Kodaira-Spencer's method, the main task in the construction of the semi-universal family is to solve

$$
\bar{\partial} f_{\mu}(t)-F \phi_{\mu}(t)=p_{\mu}(t)
$$

where $p_{\mu}(t)$ is the $\mu$ th homogeneous term of $\phi^{(\mu-1)}(t) f^{(\mu-1)}(t)$ and $F=d f_{0}$ : $T^{1,0} U \rightarrow T^{1,0} \mathbb{C}_{\mid U}^{N}$. Under some cohomological assumption, we can solve it by $R f_{\mu}(t)=\bar{\partial}^{*} N R p_{\mu}(t)$ and $F \phi_{\mu}(t)=-p_{\mu}(t)-\bar{\partial} f_{\mu}(t)$ where $R: T^{1,0} \mathbb{C}_{\mid U}^{N} \rightarrow N_{U / \mathbb{C}^{N}}$ denotes the quotient map and $N$ denotes the Neumann operator on $A_{\bar{\Omega}}^{0,1}\left(N_{U / \mathbb{C}^{N}}\right)$. Since we have $\left\|\bar{\partial}^{*} N R p_{\mu}\right\|_{(0, k)}^{\prime}(t) \ll C\left\|p_{\mu}\right\|_{(0, k)}(t),\left\|f_{\mu}\right\|_{(0, k)}^{\prime}(t)$ and $\left\|\phi_{\mu}\right\|_{(0, k)}(t)$ are estimated by $\left\|p_{\mu}\right\|_{(0, k)}(t)$. However, in this case, the loss of the differentiability in the estimate $\|\phi f\|_{(0, k)} \leq C\|\phi\|_{\left(\frac{1}{2}, k\right)}\|f\|_{(0, k)}^{\prime}$ (under the condition that $\phi_{\mid \partial \Omega}$ is ${ }^{\circ} \tilde{T}^{\prime} U$ valued) is hard to be taken in anywhere. We remark that this analytical difficulty does not appear in the CR-approach if we use the Folland-Stein norm $\|\cdot\|_{(k)}$ (cf. [Mi3]).

In this paper, we employ a fine analysis on the estimate for the Neumann operator in [BGS] and [GS] where the following sharp estimate for the Neumann operator is established with respect to the standard Sobolev norm on a bounded domain $\Omega$ satisfying the $Z$ (1)-condition:

$$
\left\|X \bar{\partial}^{*} N u\right\|_{k} \leq C\|u\|_{k} \quad \text { for } u \in A_{\bar{\Omega}}^{0,1} \text { and } X \in \Gamma\left(\bar{\Omega}, T^{1,0} U\right)
$$


if the vector field $X$ lies in $\mathbb{C} T \partial \Omega$ on $\partial \Omega$. (This estimate can be generalized for $u \in A_{\bar{\Omega}}^{0,1}(E)$ with a holomorphic vector bundle $E$ on a resolution $\widetilde{V}$ of $V$.)

Using this sharp estimate, we will have

$$
\begin{aligned}
\left\|\phi_{\mu}\right\|_{k}(t)+\sum_{X}\left\|X f_{\mu}\right\|_{k}(t) & \ll C\left\|p_{\mu}\right\|_{k}(t) \\
& \ll C^{\prime}\left\|\phi^{(\mu-1)}\right\|_{k}(t) \sum_{X}\left\|X f^{(\mu-1)}\right\|_{k}(t)
\end{aligned}
$$

if $\phi^{(\mu-1)}(t)_{\mid \partial \Omega}$ is $T^{1,0} U_{\mid \partial \Omega} \cap \mathbb{C} T \partial \Omega$-valued, where $X$ runs over $(1,0)$-vector fields appearing in the coefficients of $\phi(t)=\lim _{\mu \rightarrow+\infty} \phi^{(\mu)}(t)$ and satisfying $X_{\mid \partial \Omega} \in$ $T^{1,0} U_{\mid \partial \Omega} \cap \mathbb{C} T \partial \Omega$. Since this estimate does not make sense if the $X$ in the above sum are infinitely many, we will show that the coefficients of $\phi(t)$ are generated by finitely many such $X$, by establishing a canonical expression of $\phi^{(\mu)}(t)$ (cf. Proposition 3.9). Thus, we can carry out the Kodaira-Spencer's step-by-step construction (improved by the Grauert division theorem) in our case and prove the first half of the Main Theorem. The proof of the latter half is parallel to the CR-case. Throughout this paper, we will assume $\operatorname{dim}_{\mathbb{C}} V \geq 3$. For the surface singularity case, our argument encounters a difficulty arising from the gap between $H^{q}\left(K_{\bar{\Omega}}^{\bullet, \bullet}\right)$ and $H^{q}\left(K_{\Omega}^{\bullet \bullet \bullet}\right)\left(\simeq \operatorname{Ext}^{q}\left(\Omega_{V}^{1}, \mathcal{O}_{V}\right)\right)$ for $q=1,2$.

The arrangement of this paper is as follows. In Section 1, we describe the infinitesimal part of the flat deformation theory of normal isolated singularities in terms of cohomologies on its regular part, and compare them with the stably embeddable deformation of complex structure on $\bar{\Omega}$. We introduce its deformation complex in the latter half of Section 1. In Section 2, we establish the homotopy formula of our deformation complex at degrees one and two, and construct formally the Kuranishi semi-universal family. In Section 3, we make some adjustments of that formal construction and prove the convergence of the formal family constructed using those adjustments.

\section{Stably embeddable deformation of complex structure}

Let $U$ be a complex manifold embedded in $\mathbb{C}^{N}$ and $\Omega \subset U$ a bounded subdomain. First, we recall the formulation of stably embeddable deformation of a neighbourhood of $\bar{\Omega}$ as a complex manifold germ along $\bar{\Omega}$.

Definition 1.1. A family of deformations of a neighbourhood of $\bar{\Omega}$ that is stably embeddable in $\mathbb{C}^{N}$ (for simplicity, we call it a family of stably embeddable 
deformation of a neighbourhood of $\bar{\Omega}$ ) is a smooth holomorphic map $\pi: \mathcal{X} \rightarrow T$ together with an embedding $\Phi: \mathcal{X} \rightarrow \mathbb{C}^{N} \times T$ such that:

(1) $\pi=p_{T} \circ \Phi$;

(2) $\mathcal{X}_{0}:=\pi^{-1}(0)(0 \in T)$ is a neighbourhood of $\bar{\Omega}$ and $\Phi_{\mid \mathcal{X}_{0}}: \mathcal{X}_{0} \rightarrow \mathbb{C}^{N}$ is a natural embedding.

Two families $\left(\mathcal{X}_{i}, \pi_{i}, \Phi_{i}\right)(i=1,2)$, parametrized by $(T, 0)$, are isomorphic if there exists an isomorphism $\chi: \mathcal{X}_{1}^{\prime} \simeq \mathcal{X}_{2}^{\prime}$ of neighbourhoods $\mathcal{X}_{i}^{\prime}$ of $\bar{\Omega}\left(\subset \pi_{i}^{-1}(0)\right)$ in $\mathcal{X}_{i}$ such that $\pi_{1}=\pi_{2} \circ \chi$ and $\chi_{\mid\left(\mathcal{X}_{1}^{\prime}\right)_{0}}=\operatorname{id}_{\left(\mathcal{X}_{1}^{\prime}\right)_{0}}$ hold. (Note that we do not require that $\Phi_{1}=\Phi_{2} \circ \chi$ holds.

A family $(\mathcal{X}, \pi, \Phi)$ of stably embeddable deformation of a neighbourhood of $\bar{\Omega}$, parametrized by $(T, 0)$, is complete if for any family $\left(\mathcal{X}^{\prime}, \pi^{\prime}, \Phi^{\prime}\right)$ of stably embeddable deformation of a neighbourhood of $\bar{\Omega}$, parametrized by $\left(T^{\prime}, 0\right)$, there exists a holomorphic map $\tau: T^{\prime} \rightarrow T$ with $\tau(0)=0$ and such that $\left(\mathcal{X}^{\prime}, \pi^{\prime}\right)$ is isomorphic to the induced family $\left(\mathcal{X} \times{ }_{T} T^{\prime}, \pi \times_{T} T^{\prime}\right)$.

Let $(\mathcal{X}, \pi, \Phi)$ be a family of stably embeddable deformation of a neighbourhood of $\bar{\Omega}$, parametrized by $(T, 0)$. Then, the infinitesimal deformation of this family is represented by an element of $\lim _{\Omega^{\prime} \supset \bar{\Omega}} \operatorname{Ker}\left\{H^{1}\left(\Omega^{\prime}, \Theta_{\Omega^{\prime}}\right) \rightarrow H^{1}\left(\Omega^{\prime}, \Theta_{\mathbb{C}^{N} \mid \Omega^{\prime}}\right)\right\}$. We denote it by $\breve{\rho}_{\bar{\Omega}}(v) \quad\left(v \in T_{0} T\right)$. A family of stably embeddable deformation of a neighbourhood of $\bar{\Omega}$ is semi-universal if it is complete and the map $\breve{\rho}_{\bar{\Omega}}$ is injective.

Since our analytic tool is the harmonic theory on $\bar{\Omega}$, we formulate the theory of stably embeddable deformation of complex structures on $\bar{\Omega}$. In the following, $A_{\bar{\Omega}}^{0, q}(E)$ denotes the space of all $E$-valued $(0, q)$-forms over $\bar{\Omega}$ that are extendable differentiably across the boundary $\partial \Omega$ and $A_{k}^{0, q}(E)$ denotes its completion with respect to the Sobolev $k$-norm.

Definition 1.2. A family of stably embeddable deformation of the complex structure on $\bar{\Omega}$ is a holomorphic family of deformations of the complex structure on $\bar{\Omega}$, $\phi(t) \in A_{\bar{\Omega}}^{0,1}\left(T^{\prime} U\right)\left[\left[t_{1}, \ldots, t_{d}\right]\right]$ together with $f(t) \in A_{\bar{\Omega}}^{0}\left(T^{\prime} \mathbb{C}^{N}{ }_{\mid U}\right)\left[\left[t_{1}, \ldots, t_{d}\right]\right]$ such that:

(0) $\phi(0)=0$ and $f(0)=0$;

(1) $\phi(t) \in A_{k}^{0,1}\left(T^{\prime} U\right)\left\{t_{1}, \ldots, t_{d}\right\}$ and $f(t) \in A_{k}^{0}\left(T^{\prime} \mathbb{C}_{\mid U}^{N}\right)\left\{t_{1}, \ldots, t_{d}\right\}$ hold for all $k \gg 0$;

(2) $\quad P(\phi(t), f(t)) \in \mathfrak{I}_{T}\left(A_{k-1}^{0,2}\left(T^{\prime} U\right) \oplus A_{k-1}^{0,1}\left(T^{\prime} \mathbb{C}_{\mid U}^{N}\right)\right)\left\{t_{1}, \ldots, t_{d}\right\}$ where we denote $P(\phi(t), f(t)):=\left(\bar{\partial} \phi(t)-\frac{1}{2}[\phi(t), \phi(t)],(\bar{\partial}-\phi(t))\left(f_{0}+f(t)\right)\right)$ with $f_{0}$ denoting the natural embedding $U \hookrightarrow \mathbb{C}^{N}$ and $\mathfrak{I} \subset \mathbb{C}\left\{t_{1}, \ldots, t_{d}\right\}$ the defining ideal of $T$. 
Definition 1.3. A family $(\phi(t), f(t))$ of stably embeddable deformation of complex structures over $\bar{\Omega}$, parametrized by $(T, 0)$, is Kuranishi versal if for any family $\left(\mathcal{X}^{\prime}, \pi^{\prime}, \Phi^{\prime}\right)$ of stably embeddable deformation of a neighbourhood of $\bar{\Omega}$, parametrized by $\left(T^{\prime}, 0\right)$, there exists a holomorphic map $\tau: T^{\prime} \rightarrow T$ with $\tau(0)=0$ and a holomorphic family of embeddings $G: \bar{\Omega} \times T^{\prime} \rightarrow \mathcal{X}^{\prime}$ such that $G_{\mid \bar{\Omega} \times 0}$ is the natural embedding $\bar{\Omega} \hookrightarrow \mathcal{X}_{0}^{\prime}$ and the family $\phi\left(\tau\left(t^{\prime}\right)\right)$ is induced from $G$ as a formal family.

For a family $(\phi(t), f(t))$ of stably embeddable deformation of complex structures over $\bar{\Omega}$, parametrized by $(T, 0)$, its infinitesimal deformation is a class in $\operatorname{Ker}\left\{H_{\bar{\partial}}^{1}\left(\bar{\Omega}, T^{\prime} U\right) \rightarrow H_{\bar{\partial}}^{1}\left(\bar{\Omega}, T^{\prime} \mathbb{C}_{\mid U}^{N}\right)\right\}$ given by $v(\phi)\left(v \in T_{0}^{\prime} T\right)$. We denote it by $\rho_{\bar{\Omega}}(v)\left(v \in T_{0} T\right)$. A family is Kuranishi semi-universal if it is Kuranishi versal and the map $\rho_{\bar{\Omega}}$ is bijective.

The subject of this paper is to consider stably embeddable deformation of complex structure on the regular part of a normal Stein space, as an analytic approach to deformation of normal isolated singularities. Let $V$ be an analytic subvariety (i.e. a reduced and irreducible analytic sub-space) of a ball $B\left(c^{*}\right):=\left\{\sum_{\beta=1}^{N}\left|w^{\beta}\right|^{2}<c^{*}\right\}$ in $\mathbb{C}^{N}$ having only one normal singularity at the origin $o$. We assume that $V$ meets spheres $\partial B(c)$ transversely for all $0<c<c^{*}$ and is defined by a finite number of holomorphic equations $h_{1}(w)=\cdots=h_{m_{1}}(w)=0$ on $B\left(c^{*}\right)$. A deformation of $V$ (parametrized by $T$ ) is a flat holomorphic map $\varpi: \mathcal{V} \rightarrow T$. We assume that $\varpi^{-1}(0) \supset \bar{V}$, there exists an embedding $\Psi: \mathcal{V} \rightarrow \mathbb{C}^{N} \times T$ satisfying $p_{T} \circ \Psi=\varpi$ with $p_{T}$ denoting the projection $\mathbb{C}^{N} \times T \rightarrow T$, and $\Psi(\mathcal{V})$ is defined by the equations $\tilde{h}_{1}(w, t)=\cdots=\tilde{h}_{m_{1}}(w, t)=0$ in $B\left(c^{*}\right) \times T$ with $\tilde{h}_{\lambda}(w, 0)=h_{\lambda}(w)(\lambda=$ $\left.1, \ldots, m_{1}\right)$.

The infinitesimal deformation of this family is represented by $\left(v\left(\tilde{h}_{1}\right), \ldots\right.$, $\left.v\left(\tilde{h}_{m_{1}}\right)\right)\left(v \in T_{0} T\right)$ as an element in $\operatorname{Ext}^{1}\left(\Omega_{V}^{1}, \mathcal{O}_{V}\right)$. We denote it by $\rho_{V}(v)$. It is described by a cohomology class on the regular part as follows. Let $G(a, b):=\{a<$ $\left.\sum_{\beta=1}^{N}\left|w^{\beta}\right|^{2}<b\right\}\left(0<a<b<c^{*}\right), \Omega_{a}^{b}:=V \cap G(a, b)$ and denote $\Omega=\Omega_{a}^{b}$. Then we have the following.

Proposition 1.1. [Mi3, Proposition 3.1] We have the following.

(1) $\operatorname{Ext}^{1}\left(\Omega_{V}^{1}, \mathcal{O}_{V}\right) \simeq \operatorname{Ker}\left\{H^{1}\left(\Omega, \Theta_{\Omega}\right) \rightarrow H^{1}\left(\Omega, \Theta_{\mathbb{C}^{N}} \otimes \mathcal{O}_{\Omega}\right)\right\}$.

(2) $\operatorname{Ext}^{2}\left(\Omega_{V}^{1}, \mathcal{O}_{V}\right) \simeq \operatorname{Ker}\left\{H^{1}\left(\Omega, N_{\Omega / \mathbb{C}^{N}}\right) \rightarrow H^{1}\left(\Omega, \mathcal{O}_{\Omega}^{m_{1}}\right)\right\}$ where $N_{\Omega / \mathbb{C}^{N}}$ denotes the normal bundle of the embedding $\Omega \hookrightarrow \mathbb{C}^{N}$. 
If we fix a $C^{\infty}$ trivialization

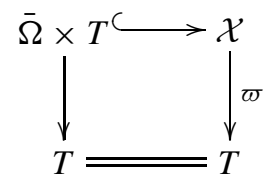

and by realizing $T$ as a subspace of a neighbourhood of the origin in some $\mathbb{C}^{d}$, we have a family $(\phi(t), f(t))$ of stably embeddable deformation of complex structures on $\bar{\Omega}$. By the same argument as [Mi3, Proposition 4.8], we have the following.

Proposition 1.2. Let $\varpi: \mathcal{V} \rightarrow T,(\mathcal{X}, \pi, \Psi)$ and $(\phi(t), f(t))$ be as above. Then, for $v \in T_{0} T, \breve{\rho}_{\bar{\Omega}}(v)$ and $-\rho_{\bar{\Omega}}(v)$ correspond to $\rho_{V}(v)$ under the isomorphisms in Proposition 1.1.

In order to carry out the construction of the Kuranishi semi-universal family, we need to introduce the deformation complex $K_{\bar{\Omega}}^{\bullet, \bullet}$, where we denote $U:=V \backslash o$ :

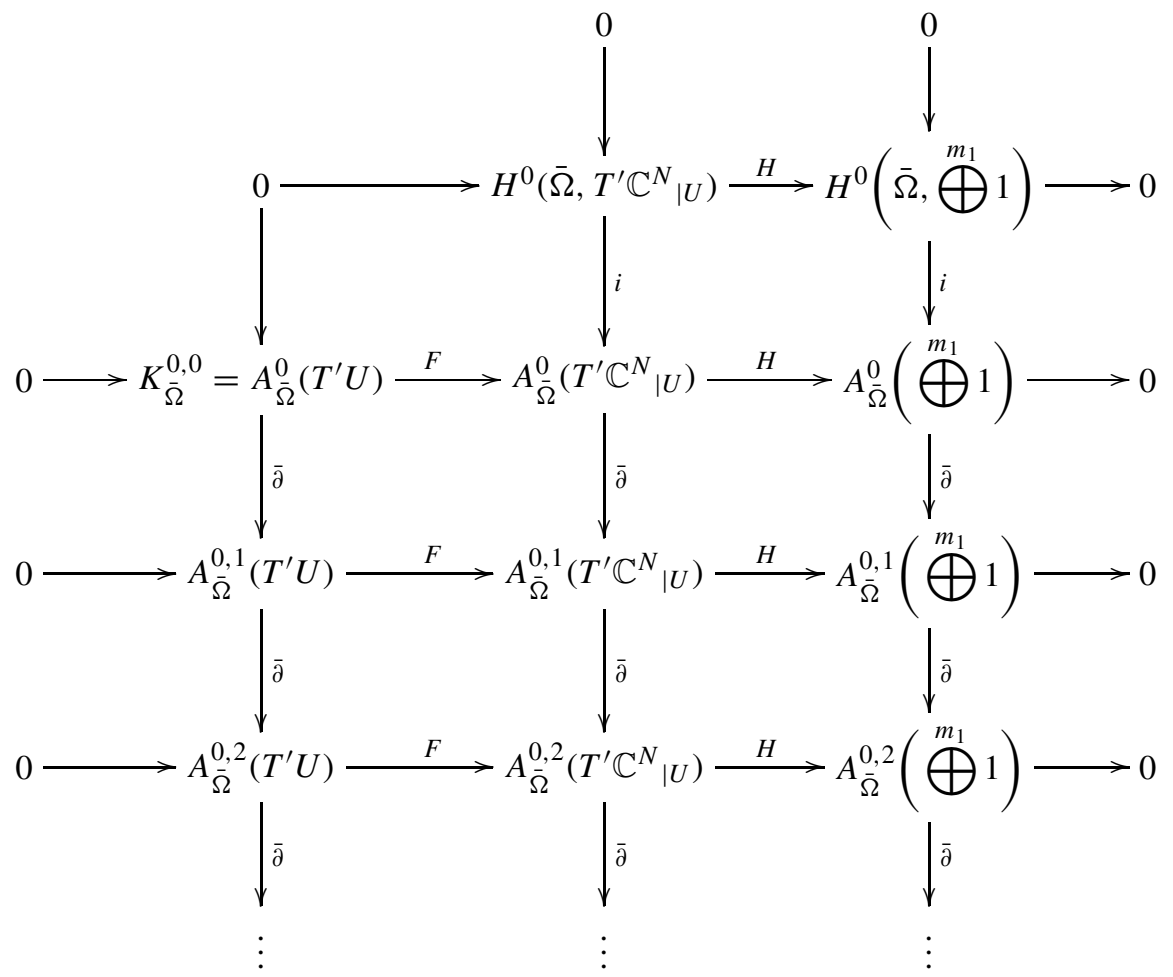


where $H$ is induced by a bundle homomorphism

$$
T^{\prime} \mathbb{C}^{N}{ }_{\mid U} \ni v \mapsto\left(v\left(h_{1}\right), \ldots, v\left(h_{m_{1}}\right)\right) \in \bigoplus^{m_{1}} 1_{U}
$$

$i$ denotes the inclusion map and we use the notation $H^{0}(\bar{\Omega}, E):=\left\{u \in A_{\bar{\Omega}}^{0,0}(E) \mid\right.$ $\bar{\partial} u=0\}$ for a holomorphic vector bundle over a neighbourhood of $\bar{\Omega}$.

We also denote by $K_{\Omega}^{\bullet, \bullet}$ the above double complex with $\bar{\Omega}$ replaced by $\Omega$. Then, the following analytic description of the Ext-groups was obtained in [Mi3, Proposition 4.5].

Proposition 1.3. For $q=1,2$,

$$
\operatorname{Ext}^{q}\left(\Omega_{V}^{1}, \mathcal{O}_{V}\right) \simeq H^{q}\left(K_{\Omega_{a}^{b}}^{\bullet \bullet \bullet}\right) \simeq \lim _{a \rightarrow b-0} H^{q}\left(K_{\overline{\Omega_{a}^{b}}}^{\bullet \bullet \bullet}\right)
$$

We infer the following from Proposition 1.3 together with [Ho, Theorems 3.4.6 and 3.4.9].

COROLlary 1.4. If $\operatorname{dim}_{\mathbb{C}} V \geq 3$, then

$$
\operatorname{Ext}^{q}\left(\Omega_{V}^{1}, \mathcal{O}_{V}\right) \simeq H^{q}\left(K_{\bar{\Omega}}^{\bullet, \bullet}\right) \quad(q=1,2) .
$$

Remark 1.5. For the surface singularity case, $H^{q}\left(K_{\bar{\Omega}}^{\bullet, \bullet}\right)$ may be infinite dimensional in general. However, if $V$ is complete intersection, $H^{2}\left(K_{\bar{\Omega}}^{\bullet, \bullet}\right)=H^{2}\left(K_{\Omega}^{\bullet, \bullet}\right)=0$ holds since $K_{\bar{\Omega}}^{1, q} \rightarrow K_{\bar{\Omega}}^{2, q}$ (respectively $K_{\Omega}^{1, q} \rightarrow K_{\Omega}^{2, q}$ ) is surjective for $q \geq 0$.

\section{Homotopy formula and a formal construction of the Kuranishi versal family}

Let $\left(K_{\bar{\Omega}}^{\bullet}, d\right)$ be the total simple complex of the double complex $K_{\bar{\Omega}}^{\bullet \cdot \bullet}$. We establish the homotopy formula for the complex $\left(K_{\bar{\Omega}}^{\bullet}, d\right)$.

THEOREM 2.1. For $q=1,2$, there exist operators $Z_{q}: K_{\bar{\Omega}}^{q} \rightarrow \operatorname{Ker} d \cap K_{\bar{\Omega}}^{q}$ and $Q_{q}: \operatorname{Ker} d \cap K_{\bar{\Omega}}^{q} \rightarrow K_{\bar{\Omega}}^{q-1}$ satisfying:

(1) $Z_{q \mid \operatorname{Ker} d}=\operatorname{id}_{\mid \operatorname{Ker} d}$;

(2) $d \circ Q_{q} \circ d=d$.

If we obtain $Z_{q}$ and $Q_{q}$ as above, we have the following homotopy formula.

COROLlARY 2.2. Under the assumption of Theorem 2.1 , if we set $\rho_{\mathcal{H}}:=(1-d \circ$ $\left.Q_{q}\right) \circ Z_{q}$ and $\mathcal{H}^{q}:=\rho_{\mathcal{H}}\left(K_{\bar{\Omega}}^{q}\right)$, then we have:

(1) the natural homomorphism $\mathcal{H}^{q} \rightarrow H^{q}\left(K_{\bar{\Omega}}^{\bullet, \bullet}\right)$ is an isomorphism;

(2) a homotopy formula $u=\rho_{\mathcal{H}} u+d \circ Q_{q} \circ Z_{q} u+\left(1-Z_{q}\right) u$ holds for $u \in K_{\bar{\Omega}}^{q}$. 
Since we use only $Z_{2}$ and $Q_{2}$ in the argument of this paper and its construction is parallel to [Mi3, Section 5], we only recall it for the case of $q=2$.

Over $U$, we have exact sequences of holomorphic vector bundles;

$$
\begin{aligned}
0 & \rightarrow T^{\prime} U \stackrel{F}{\rightarrow} T^{\prime} \mathbb{C}_{\mid U}^{N} \stackrel{R}{\rightarrow} N_{U / \mathbb{C}^{N}} \rightarrow 0, \\
0 & \rightarrow N_{U / \mathbb{C}^{N}} \stackrel{i}{\rightarrow} \stackrel{m_{1}}{\bigoplus} 1 \stackrel{s}{\rightarrow} E \rightarrow 0
\end{aligned}
$$

where $N_{U / \mathbb{C}^{N}}$ denotes the normal bundle of the embedding $\iota: U \hookrightarrow \mathbb{C}^{N}$ and $E$ the holomorphic vector bundle $\bigoplus^{m_{1}} 1 / N_{U / \mathbb{C}^{N}}$ over $U$. Note that $H=i \circ R$. We fix a differentiable projection $p$ and a differentiable splitting $q$ :

$$
0 \rightarrow T^{\prime} U \underset{p}{\stackrel{F}{\longrightarrow}} T^{\prime} \mathbb{C}_{\mid U}^{N} \underset{q}{\stackrel{R}{\rightleftarrows}} N_{U / \mathbb{C}^{N}} \rightarrow 0
$$

We fix the Hodge-Kodaira decompositions $u=\rho u+\square N u$ for $u \in A_{\bar{\Omega}}^{0,1}\left(N_{U / \mathbb{C}^{N}}\right)$ or $u \in A_{\bar{\Omega}}^{0,1}$ and $v=\rho v+\bar{\partial}^{*} N \bar{\partial} v$ for $v \in A_{\bar{\Omega}}^{0}$. Let $\mathbf{H}_{N_{U / \mathbb{C}}^{N}}^{1}:=\{u \in \operatorname{Dom}(\square) \subset$ $\left.A_{\bar{\Omega}}^{0,1}\left(N_{U / \mathbb{C}^{N}}\right) \mid \square u=0\right\}$ and $\mathbf{H}^{1}:=\left\{u \in \operatorname{Dom}(\square) \subset A_{\bar{\Omega}}^{0,1} \mid \square u=0\right\}$. Consider the homomorphism $\bigoplus^{m_{1}} \rho \circ i_{\mid \mathbf{H}_{N_{U / \mathbb{C}}}^{1}}: \mathbf{H}_{N_{U / \mathbb{C}^{N}}}^{1} \rightarrow \bigoplus^{m_{1}} \mathbf{H}^{1}$ and denote its kernel by $\mathbf{H}$. We fix a projection $\rho_{\mathbf{H}}: \mathbf{H}_{N_{U / \mathbb{C}}^{N}}^{1} \rightarrow \mathbf{H}$. We set $H^{1}:=q(\mathbf{H}) \subset A_{\bar{\Omega}}^{0,1}\left(T^{\prime} \mathbb{C}_{\mid U}^{N}\right)$.

$$
\begin{aligned}
& \text { For }\left(a_{2}, b_{1}, c_{0}\right) \in K_{\bar{\Omega}}^{0,2} \oplus K_{\bar{\Omega}}^{1,1} \oplus K_{\bar{\Omega}}^{2,0}, \text { let } \\
& \qquad \check{b}_{1}:=q \circ\left(\rho_{\mathbf{H}} \circ \rho_{N}+\bar{\partial} \circ \bar{\partial}^{*} \circ N\right) \circ R\left(b_{1}\right) .
\end{aligned}
$$

Then, $Z_{2}$ is given by the formula;

$$
Z_{2}\left(a_{2}, b_{1}, c_{0}\right):=\left(a_{2}^{\prime}, b_{1}^{\prime}, c_{0}^{\prime}\right)
$$

where

$$
\begin{aligned}
& b_{1}^{\prime}:=\breve{b}_{1}+p\left(b_{1}-\check{b}_{1}\right) \\
& a_{2}^{\prime}:=-p \bar{\partial}\left(b_{1}^{\prime}\right) \\
& c_{0}^{\prime}:=\bar{\partial}^{*} \circ N \circ H\left(\check{b}_{1}\right)+\rho\left(c_{0}\right) .
\end{aligned}
$$

Next, for $\left(a_{2}^{\prime}, b_{1}^{\prime}, c_{0}^{\prime}\right) \in \operatorname{Ker} d \subset K_{\bar{\Omega}}^{0,2} \oplus K_{\bar{\Omega}}^{1,1} \oplus K_{\bar{\Omega}}^{2,0}$,

$$
Q_{2}\left(a_{2}^{\prime}, b_{1}^{\prime}, c_{0}^{\prime}\right):=\left(a_{1}^{\prime \prime}, b_{0}^{\prime \prime}, c_{-1}^{\prime \prime}\right)
$$


where

$$
\begin{aligned}
b_{0}^{\prime \prime} & :=q \circ \bar{\partial}^{*} \circ N \circ R\left(b_{1}^{\prime}\right) \\
a_{1}^{\prime \prime} & :=-\left(b_{1}^{\prime}-q \circ \rho \circ R\left(b_{1}^{\prime}\right)-\bar{\partial}\left(b_{0}^{\prime \prime}\right)\right) \\
c_{-1}^{\prime \prime} & :=c_{0}^{\prime}-H\left(b_{0}^{\prime \prime}\right)-\bar{\partial}^{*} \circ N \circ i \circ \rho \circ R\left(b_{1}^{\prime}\right) .
\end{aligned}
$$

In order for the construction of the Kuranishi semi-universal family, we need the estimates for these operators with respect to the standard Sobolev norm $\|\cdot\|_{k}$.

First, we summarize fundamental estimates for the Neumann operator $N$.

PROPOSITION 2.3. Let $E$ be a holomorphic vector bundle over $U$ and $N$ : $A_{\bar{\Omega}}^{0,1}(E) \rightarrow A_{\bar{\Omega}}^{0,1}(E)$ denotes the Neumann operator. Let $U_{1}$ be a coordinate neighbourhood on which $E$ is trivialized and let $\varphi \in C_{0}^{\infty}\left(U_{1}\right)$.

(1) There exists $C>0$ such that $\|\varphi N u\|_{k+1} \leq C\|u\|_{k}$ holds for $u \in A_{\bar{\Omega}}^{0,1}(E)$.

(2) Suppose that $X, Y \in \Gamma(\bar{\Omega}, \mathbb{C} T U)$ and $X$ and $Y$ lie in ${ }^{\circ} T_{x}^{\prime} \partial \Omega \oplus^{\circ} T_{x}^{\prime \prime} \partial \Omega$ for all $x \in \partial \Omega$. Then, there exists $C^{\prime}>0$ such that $\|X Y \varphi N u\|_{k} \leq C^{\prime}\|u\|_{k}$ and $\left\|X \varphi \bar{\partial}^{*} N u\right\|_{k} \leq C^{\prime}\|u\|_{k}$ hold for $u \in A_{\bar{\Omega}}^{0,1}(E)$.

The estimate (1) is standard. The sharp estimate (2) is proved in [BGS] and [GS] for the Neumann operator for the complex $A_{\bar{\Omega}}^{p, \bullet}$. The argument there also works for the vector bundle-valued $\bar{\partial}$-complex $A_{\bar{\Omega}}^{0, \bullet}(E)$ since the main technique is based on the calculus of local solutions for the Laplace-Beltrami equation satisfying the Neumann boundary condition.

The following estimates follow from the definition of $Z_{2}$ and $Q_{2}$ together with Proposition 2.3.

Proposition 2.4. For $\left(a_{2}, b_{1}, c_{0}\right) \in K_{\bar{\Omega}}^{0,2} \oplus K_{\bar{\Omega}}^{1,1} \oplus K_{\bar{\Omega}}^{2,0}$, let $Z_{2}\left(a_{2}, b_{1}, c_{0}\right)=$ $\left(a_{2}^{\prime}, b_{1}^{\prime}, c_{0}^{\prime}\right)$ and $Q_{2}\left(a_{2}^{\prime}, b_{1}^{\prime}, c_{0}^{\prime}\right)=\left(a_{1}^{\prime \prime}, b_{0}^{\prime \prime}, c_{-1}^{\prime \prime}\right)$. Then:

(1) there exists $C>0$ such that

$$
\frac{1}{C}\left(\left\|a_{1}^{\prime \prime}\right\|_{k}+\left\|b_{0}^{\prime \prime}\right\|_{k}\right) \leq\left\|b_{1}^{\prime}\right\|_{k} \leq C\left\|b_{1}\right\|_{k}
$$

(2) if $X$ is as in Proposition 2.3, then there exists $C^{\prime}>0$ such that

$$
\left\|X b_{0}^{\prime \prime}\right\|_{k} \leq C^{\prime}\left\|b_{1}^{\prime}\right\|_{k} .
$$

Remark 2.5. Note that the constants $C$ and $C^{\prime}$ in Propositions 2.3 and 2.4 depend on the vector fields $X$ and $Y$. 
In the rest of this section, we construct the Kuranishi semi-universal family of stably embeddable deformation of complex structure on $\bar{\Omega}$ as a formal power series of the parameters.

THEOREM 2.6. There exist a family $(\phi(t), f(t), k(t)) \in K_{\bar{\Omega}}^{1}\left[\left[t_{1}, \ldots, t_{d}\right]\right]$ and an ideal $\mathfrak{I} \subset \mathbb{C}\left[\left[t_{1}, \ldots, t_{d}\right]\right]$ such that:

(1) $\quad(\phi(0), f(0), k(0))=(0,0,0)$;

(2) $\quad(\phi(t), f(t), k(t)) \equiv \sum_{\alpha=1}^{d}\left(\phi_{\alpha}, f_{\alpha}, k_{\alpha}\right) t_{\alpha} \bmod \mathfrak{m}^{2}$ where $\left\{\left[\left(\phi_{\alpha}, f_{\alpha}, k_{\alpha}\right)\right]\right\}_{\alpha=1}^{d}$ forms a cohomology basis of $H^{1}\left(K_{\overline{\mathbf{\Omega}}}^{\bullet \bullet \bullet}\right)$ and $\mathfrak{m}$ denotes the maximal ideal of $\mathbb{C}\left\{t_{1}, \ldots, t_{d}\right\}$;

(3) there exists an extension $\tilde{k}(t) \in H^{0}\left(\bar{B}(b), \mathcal{O}_{\mathbb{C}^{N}}\right)\left[\left[t_{1}, \ldots, t_{d}\right]\right]$ of $k(t)$ such that

$$
\begin{aligned}
& P(\phi(t), f(t), \tilde{k}(t)) \\
& :=\left(\bar{\partial} \phi(t)-\frac{1}{2}[\phi(t), \phi(t)],(\bar{\partial}-\phi(t))\left(f_{0}+f(t)\right),(\tilde{h}+\tilde{k}(t)) \circ\left(f_{0}+f(t)\right)\right) \\
& \in \Im K_{\bar{\Omega}}^{2}\left[\left[t_{1}, \ldots, t_{d}\right]\right] ;
\end{aligned}
$$

(4) $\mathfrak{I}$ is generated by $b_{1}(t), \ldots, b_{\ell}(t)$ where

$$
\rho_{\mathcal{H}} P(\phi(t), f(t), \tilde{k}(t))=\sum_{\beta=1}^{\ell} b_{\beta}(t) e_{\beta}
$$

for a fixed basis $e_{1}, \ldots, e_{\ell}$ of $\mathcal{H}^{2}$.

We construct $(\phi(t), f(t), k(t))$ by the Kodaira-Spencer's step-by-step construction improved by the Grauert division theorem (cf. [Mi3]). We denote by $\phi^{(\mu)}(t)$ (respectively $f^{(\mu)}(t)$ or $\left.k^{(\mu)}(t)\right)$ a $K_{\bar{\Omega}}^{0,1}\left(\right.$ respectively $K_{\bar{\Omega}}^{1,0}$ or $K_{\bar{\Omega}}^{2,-1}$ ) valued polynomial of $\left(t_{1}, \ldots, t_{d}\right)$ of degree $\mu$.

By (1) and (2), let $\left(\phi^{(1)}(t), f^{(1)}(t), k^{(1)}(t)\right):=\sum_{\alpha=1}^{d}\left(\phi_{\alpha}, f_{\alpha}, k_{\alpha}\right) t_{\alpha}$ where we choose a $d$-closed element $\left(\phi_{\alpha}, f_{\alpha}, k_{\alpha}\right) \in K_{\bar{\Omega}}^{1}$ such that $\left\{\left[\left(\phi_{\alpha}, f_{\alpha}, k_{\alpha}\right)\right]\right\}_{\alpha=1, \ldots, d}$ is a system of cohomology basis of $H^{1}\left(K_{\bar{\Omega}}^{\bullet \bullet \bullet}\right)$. Let $\tilde{k}_{\alpha}$ be a holomorphic extension of $k_{\alpha}$ over $\overline{B(b)}$. We remark that such holomorphic extension is possible since $V$ is a normal subvariety (cf. [BE, Proposition 2.1] or [Ad]).

We suppose that $\left(\phi^{(\mu-1)}(t), f^{(\mu-1)}(t), k^{(\mu-1)}(t)\right)$ and an extension $\tilde{k}^{(\mu-1)}(t) \in$ $\left.H^{0} \overline{(B(b)}, \bigoplus^{m_{1}} \mathcal{O}_{\mathbb{C}^{N}}\right)\left[t_{1}, \ldots, t_{d}\right]$ of $k^{(\mu-1)}(t)$ are determined.

Let $\left(\phi_{\mu}(t), f_{\mu}(t), k_{\mu}(t)\right)$ denote the $\mu$ th homogeneous term of $-Q_{2} \circ Z_{2} \circ$ $P\left(\phi^{(\mu-1)}(t), f^{(\mu-1)}(t), \tilde{k}^{(\mu-1)}(t)\right)$ and denote

$$
\begin{aligned}
& \left(\phi^{(\mu)}(t), f^{(\mu)}(t), k^{(\mu)}(t)\right) \\
& \quad:=\left(\phi^{(\mu-1)}(t), f^{(\mu-1)}(t), k^{(\mu-1)}(t)\right)+\left(\phi_{\mu}(t), f_{\mu}(t), k_{\mu}(t)\right) .
\end{aligned}
$$


If we let

$(\phi(t), f(t), k(t)):=\lim _{\mu \rightarrow+\infty}\left(\phi^{(\mu)}(t), f^{(\mu)}(t), k^{(\mu)}(t)\right) \in K_{\bar{\Omega}}^{1}\left[\left[t_{1}, \ldots, t_{d}\right]\right]$

and

$$
\tilde{k}(t):=\lim _{\mu \rightarrow+\infty} \tilde{k}^{(\mu)}(t) \in H^{0}\left(\overline{B(b)}, \bigoplus^{m_{1}} \mathcal{O}_{\mathbb{C}^{N}}\right)\left[\left[t_{1}, \ldots, t_{d}\right]\right],
$$

Theorem 2.6 is proved by the same argument as the first half of [Mi3, Section 8].

\section{Adjustment of the formal solution and its convergence}

In Section 2, we constructed a formal family $(\phi(t), f(t), k(t)) \in K_{\bar{\Omega}}^{1}\left[\left[t_{1}, \ldots, t_{d}\right]\right]$. In order to assure the convergence of $\phi(t)$ and $f(t)$, we need to require an additional condition that $\phi(t)_{\mid \partial \Omega}$ is $T^{\prime} U_{\mid \partial \Omega} \cap \mathbb{C} T \partial \Omega$-valued. In this section, we make three adjustments at each step of constructing a finite-order solution $\left(\phi^{(\mu)}(t), f^{(\mu)}(t), k^{(\mu)}(t)\right)$ so that the resulting solution $\phi(t)$ fulfills this requirement. Let

$$
\begin{gathered}
\mathcal{K}^{0, q}:=\left\{a_{q} \in A_{\bar{\Omega}}^{0, q}\left(T^{1,0} U\right) \mid\left(a_{q}\right)_{\mid \partial \Omega} \text { is }\left(T^{\prime} U_{\mid \partial \Omega} \cap \mathbb{C} T \partial \Omega\right) \text {-valued }\right\} \\
\mathcal{K}^{1, q}:=\left\{b_{q} \in A_{\bar{\Omega}}^{0, q}\left(T^{1,0} \mathbb{C}^{N} \mid U\right) \mid\left(b_{q}\right)_{\mid \partial \Omega} \text { is }\left(T^{\prime} \mathbb{C}^{N} \cap \mathbb{C} T S^{2 N-1}\right)_{\mid \partial \Omega \text {-valued }\} .}\right.
\end{gathered}
$$

Then, the purpose of this section is to establish a canonical way to construct a solution $(\phi(t), f(t), k(t))$ belonging to $\left(\mathcal{K}^{0,1} \oplus \mathcal{K}^{1,0} \oplus K_{\bar{\Omega}}^{2,-1}\right)\left[\left[t_{1}, \ldots, t_{d}\right]\right]$.

We use the same notation as in [Mi3] about the CR structure on the boundary $M=$ $\partial \Omega$ : Let ${ }^{\circ} T^{\prime}:=T^{\prime} U_{\mid M} \cap \mathbb{C} T M$ and ${ }^{\circ} T^{\prime \prime}:={ }^{\circ} T^{\prime}$. For a differentiable vector bundle $E_{b}$ over $M, A_{b}^{0, q}\left(E_{b}\right):=\Gamma\left(M, E_{b} \otimes \wedge^{q}\left({ }^{\circ} T^{\prime \prime}\right)^{*}\right)$. For a differentiable vector bundle $E$ over $U, \tau: A_{\bar{\Omega}}^{0, q}(E) \rightarrow A_{b}^{0, q}\left(E_{b}\right)$ is defined by $\tau(\phi)\left(u_{1}, \ldots, u_{q}\right):=\phi\left(u_{1}, \ldots, u_{q}\right)$ for $u_{1}, \ldots, u_{q} \in \Gamma\left(M,{ }^{\circ} T^{\prime \prime}\right)$. In particular, the analytic restriction $\tau: A_{\bar{\Omega}}^{0}(E) \rightarrow A_{b}^{0}\left(E_{b}\right)$ coincides with the set theoretical restriction.

We denote $r:=\sum_{\beta=1}^{N}\left|w^{\beta}\right|^{2}$ and let ${ }^{\circ} \tilde{T}^{\prime} \mathbb{C}^{N}:=\left\{u \in T^{\prime} \mathbb{C}^{N} \mid u(r)=0\right\}$ and ${ }^{\circ} \tilde{T}^{\prime} U:=\left\{u \in T^{\prime} U \mid u(r)=0\right\}$. Then ${ }^{\circ} \tilde{T}^{\prime} \mathbb{C}^{N}$ (respectively ${ }^{\circ} \tilde{T}^{\prime} U$ ) is a differentiable vector subbundle of $T^{\prime} \mathbb{C}^{N}$ over $\mathbb{C}^{N} \backslash o$ (respectively of $T^{\prime} U$ over $U$ ). We fix a vector field $\eta$ on $U$ complementary to ${ }^{\circ} \tilde{T}^{\prime} U$ and $\xi:=\sum_{\beta=1}^{N} w^{\beta}\left(\partial / \partial w^{\beta}\right)$. Then we have the splittings as differentiable vector bundles;

$$
\begin{aligned}
T^{\prime} \mathbb{C}^{N} \mid \mathbb{C}^{N} \backslash o & =\mathbb{C} \xi \oplus^{\circ} \tilde{T}^{\prime} \mathbb{C}^{N} \mid \mathbb{C}^{N} \backslash o, \\
T^{\prime} U & =\mathbb{C} \eta \oplus^{\circ} \tilde{T}^{\prime} U .
\end{aligned}
$$

Then we have the following. 
PROPOSITION 3.1. There exists a differentiable splitting

$$
0 \rightarrow T^{\prime} U \underset{\leftarrow}{\stackrel{F}{\rightarrow}} T^{\prime} \mathbb{C}^{N} \mid U \underset{\leftarrow}{\stackrel{R}{\rightarrow}} N_{U / \mathbb{C}^{N}} \rightarrow 0
$$

such that

$$
p\left({ }^{\circ} \tilde{T}^{\prime} \mathbb{C}^{N}{ }_{\mid U}\right) \subset{ }^{\circ} \tilde{T}^{\prime} U \text { and } q\left(N_{U / \mathbb{C}^{N}}\right) \subset{ }^{\circ} \tilde{T}^{\prime} \mathbb{C}^{N} \mid U .
$$

The proof is parallel to [Mi3, Proposition 6.3].

Hence, we have a similar setting as in the CR-case and the first adjustment is done by constructing $Z_{2}$ and $Q_{2}$ as in Section 2 relying on the splitting (3.2). Then, it is clear that $b_{0}^{\prime \prime} \in A_{\bar{\Omega}}^{0}\left({ }^{\circ} \tilde{T}^{\prime} \mathbb{C}^{N}{ }_{\mid U}\right)$ holds if $\left(a_{1}^{\prime \prime}, b_{0}^{\prime \prime}, c_{-1}^{\prime \prime}\right)=Q_{2}\left(a_{2}^{\prime}, b_{1}^{\prime}, c_{0}^{\prime}\right)$.

Denote $p_{\xi}: T^{\prime} \mathbb{C}^{N} \mid \mathbb{C}^{N} \backslash o \rightarrow \mathbb{C} \xi$ and $p_{\eta}: T^{\prime} U \rightarrow \mathbb{C} \eta$ the projections with respect to the splitting (3.1) and by $\tilde{\tau}:\left(T^{\prime \prime} U\right)^{*} \rightarrow\left({ }^{\circ} \tilde{T}^{\prime \prime} U\right)^{*}$ the restriction operator. Let, for $q \geq 1$,

$$
\begin{aligned}
T^{\prime} \mathbb{C}^{N} \mid U \otimes \wedge^{q}\left(T^{\prime \prime} U\right)^{*}= & { }^{\circ} \tilde{T}^{\prime} \mathbb{C}^{N} \mid U \otimes \wedge^{q}\left(T^{\prime \prime} U\right)^{*}+\mathbb{C} \xi \otimes \wedge^{q}\left({ }^{\circ} \tilde{T}^{\prime \prime} U\right)^{*} \\
& +\mathbb{C} \xi \otimes \wedge^{q-1}\left({ }^{\circ} \tilde{T}^{\prime \prime} U\right)^{*} \otimes(\mathbb{C} \bar{\eta})^{*}, \\
T^{\prime} U \otimes \wedge^{q}\left(T^{\prime \prime} U\right)^{*}= & { }^{\circ} \tilde{T}^{\prime} U \otimes \wedge^{q}\left(T^{\prime \prime} U\right)^{*}+\mathbb{C} \eta \otimes \wedge^{q}\left({ }^{\circ} \tilde{T}^{\prime \prime} U\right)^{*} \\
& +\mathbb{C} \eta \otimes \wedge^{q-1}\left({ }^{\circ} \tilde{T}^{\prime \prime} U\right)^{*} \otimes(\mathbb{C} \bar{\eta})^{*}
\end{aligned}
$$

be decompositions according to the splitting (3.1). We denote by $\tilde{p}_{0}, \tilde{p}_{10}$ and $\tilde{p}_{11}$ the projections of $A_{\bar{\Omega}}^{0, q}\left(T^{\prime} \mathbb{C}^{N} \mid U\right)$ to $\Gamma\left(\bar{\Omega},{ }^{\circ} \tilde{T}^{\prime} \mathbb{C}^{N} \mid U \otimes \wedge^{q}\left(T^{\prime \prime} U\right)^{*}\right), \Gamma(\bar{\Omega}, \mathbb{C} \xi \otimes$ $\left.\wedge^{q}\left({ }^{\circ} \tilde{T}^{\prime \prime} U\right)^{*}\right)$ and $\Gamma\left(\bar{\Omega}, \mathbb{C} \xi \otimes \wedge^{q-1}\left({ }^{\circ} \tilde{T}^{\prime \prime} U\right)^{*} \otimes(\mathbb{C} \bar{\eta})^{*}\right)$, respectively, according to the decomposition (3.3), and we use the same symbols $\tilde{p}_{0}, \tilde{p}_{10}$ and $\tilde{p}_{11}$ for the projections of $A_{\bar{\Omega}}^{0, q}\left(T^{\prime} U\right)$ onto the each factors. We remark that $\phi$ is ${ }^{\circ} \tilde{T}^{\prime} U$-valued (respectively $f$

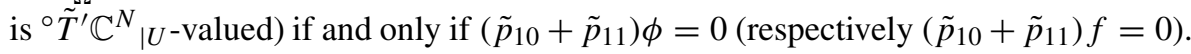
$\tau \phi$ is ${ }^{\circ} T^{\prime} \partial \Omega$-valued if and only if $\tilde{p}_{10} \phi_{\mid \partial \Omega}=0$, since $\tau \circ \tilde{p}_{11}=0$.

$\left(\tilde{p}_{0}+\tilde{p}_{10}\right) \circ \bar{\partial}$ has the following property similar to the $\bar{\partial}_{b}$-operator:

$\tilde{p}_{10} \circ \bar{\partial}: A_{U}^{0}\left({ }^{\circ} \tilde{T}^{\prime} \mathbb{C}^{N} \mid U\right) \rightarrow \Gamma\left(U, \mathbb{C} \xi \otimes\left({ }^{\circ} \tilde{T}^{\prime \prime} U\right)^{*}\right)$ is $C^{\infty}(U)$-linear, and

$\tilde{p}_{10} \circ \bar{\partial}: A_{U}^{0}\left({ }^{\circ} \tilde{T}^{\prime} U\right) \rightarrow \Gamma\left(U, \mathbb{C} \eta \otimes\left({ }^{\circ} \tilde{T}^{\prime \prime} U\right)^{*}\right)$ is a $C^{\infty}(U)$-isomorphism.

This enables us to apply the argument in [Mi3, Section 6] to our case in order to make the second adjustment so that $\tilde{p}_{10} \phi_{\mu}(t)=0$ holds on $\partial \Omega$. Let $\tilde{L}_{U}:=\tilde{p}_{10} \circ F \circ \bar{\partial}$ : $A_{\bar{\Omega}}^{0}\left({ }^{\circ} \tilde{T}^{\prime} U\right) \rightarrow \Gamma\left(U, \mathbb{C} \xi \otimes\left({ }^{\circ} \tilde{T}^{\prime} U\right)^{*}\right)$. Then, $\tilde{L}_{U}$ is a $C^{\infty}(U)$-isomorphism. We define an operator $A: K_{\bar{\Omega}}^{1} \rightarrow K_{\bar{\Omega}}^{1}$ by

$$
A\left(a_{1}^{\prime \prime}, b_{0}^{\prime \prime}, c_{-1}^{\prime \prime}\right)=\left(a_{1}^{\prime \prime}, b_{0}^{\prime \prime}, c_{-1}^{\prime \prime}\right)-d\left(\tilde{L}_{U}^{-1}\left(\tilde{p}_{10} \bar{\partial} b_{0}^{\prime \prime}\right), 0\right) .
$$

The following proposition is obvious by the definition of $A$. 
Proposition 3.2. Let $\left(a_{1}^{\prime \prime}, b_{0}^{\prime \prime}, c_{-1}^{\prime \prime}\right) \in K_{\bar{\Omega}}^{1}$ satisfy $b_{0}^{\prime \prime} \in A_{\bar{\Omega}}^{0}\left({ }^{\circ} \tilde{T}^{\prime} \mathbb{C}_{\mid U}^{N}\right)$. If $\left(a_{1}^{\prime \prime \prime}, b_{0}^{\prime \prime \prime}, c_{-1}^{\prime \prime \prime}\right):=A\left(a_{1}^{\prime \prime}, b_{0}^{\prime \prime}, c_{-1}^{\prime \prime}\right)$, then $b_{0}^{\prime \prime \prime} \in A_{\bar{\Omega}}^{0}\left({ }^{\circ} \tilde{T}^{\prime} \mathbb{C}_{\mid U}^{N}\right)$ and $\tilde{p}_{10}\left(\bar{\partial} b_{0}^{\prime \prime \prime}\right)=0$.

Remark 3.3. Since $b_{0}^{\prime \prime} \in A_{\bar{\Omega}}^{0}\left({ }^{\circ} \tilde{T}^{\prime} \mathbb{C}_{\mid U}^{N}\right)$ and $\tilde{L}_{U}^{-1}\left(\tilde{p}_{10} \bar{\partial} b_{0}^{\prime \prime}\right) \in A_{\bar{\Omega}}^{0}\left({ }^{\circ} \tilde{T}^{\prime} U\right)$, the assignments $b_{0}^{\prime \prime} \mapsto \tilde{L}_{U}^{-1}\left(\tilde{p}_{10} \bar{\partial} b_{0}^{\prime \prime}\right)$ and $b_{0}^{\prime \prime} \mapsto \tilde{p}_{11} F \bar{\partial} \tilde{L}_{U}^{-1}\left(\tilde{p}_{10} \bar{\partial} b_{0}^{\prime \prime}\right)$ are $C^{\infty}(\bar{\Omega})$-linear.

We make the final adjustment. We recall the following lemma, which is proved in the proof of Theorem 7.1 of [Ak2].

LeMmA 3.4. For any $a_{1} \in A_{\bar{\Omega}}^{0,1}\left(T^{\prime} U\right)$ with $\tau\left(a_{1}\right) \in A_{b}^{0,1}\left({ }^{\circ} \tilde{T}^{\prime} U_{\mid \partial \Omega}\right)$, there exists an $a_{0} \in A_{\bar{\Omega}}^{0}\left(T^{\prime} U\right)$ such that:

(1) $\left(a_{0}\right)_{\mid \partial \Omega}=0$;

(2) $\quad\left(a_{1}-\bar{\partial} a_{0}\right)_{\mid \partial \Omega}$ is ${ }^{\circ} \tilde{T}^{\prime} U_{\mid \partial \Omega}$-valued.

Let $F \eta=\gamma \xi+X$ with $\gamma \in C^{\infty}(U)$ and $X \in \Gamma\left(U,{ }^{\circ} \tilde{T}^{\prime} \mathbb{C}^{N}\right)$. Denote by $\theta \in A_{\bar{\Omega}}^{0}\left(T^{\prime} U\right)$ the $a_{0}$ in Lemma 3.4 for $a_{1}=\gamma^{-1} \eta \otimes \bar{\eta}^{*} \in A_{\bar{\Omega}}^{0}\left(T^{\prime} U\right)$ and $\theta_{1}:=\left(\gamma^{-1} \eta \otimes \bar{\eta}^{*}-\bar{\partial} \theta\right)$. Note that $\theta_{\mid \partial \Omega}=0$ and $\theta_{1 \mid \partial \Omega}$ is ${ }^{\circ} \tilde{T}^{\prime} U_{\mid \partial \Omega}$-valued.

For $\left(a_{1}^{\prime \prime \prime}, b_{0}^{\prime \prime \prime}, c_{-1}^{\prime \prime \prime}\right) \in K_{\bar{\Omega}}^{0,1} \oplus K_{\bar{\Omega}}^{1,0} \oplus K_{\bar{\Omega}}^{2,-1}$ such that $b_{0}^{\prime \prime \prime} \in A_{\bar{\Omega}}^{0}\left({ }^{\circ} \tilde{T}^{\prime} \mathbb{C}_{\mid U}^{N}\right)$ and $\tilde{p}_{10}\left(\bar{\partial} b_{0}^{\prime \prime \prime}\right)=0$, we denote $\psi \xi \otimes \bar{\eta}^{*}:=\tilde{p}_{11}\left(\bar{\partial} b_{0}^{\prime \prime \prime}\right)$. Then, let

$$
B\left(a_{1}^{\prime \prime \prime}, b_{0}^{\prime \prime \prime}, c_{-1}^{\prime \prime \prime}\right):=\left(a_{1}^{\prime \prime \prime}, b_{0}^{\prime \prime \prime}, c_{-1}^{\prime \prime \prime}\right)-d(\psi \theta, 0)
$$

The following proposition follows from the definition of $B$.

Proposition 3.5. Suppose $b_{0}^{\prime \prime \prime} \in A_{\bar{\Omega}}^{0}\left({ }^{\circ} \tilde{T}^{\prime} \mathbb{C}_{\mid U}^{N}\right)$ and $\tilde{p}_{10}\left(\bar{\partial} b_{0}^{\prime \prime \prime}\right)=0$. If we denote $\left(a_{1}^{\prime \prime \prime \prime}, b_{0}^{\prime \prime \prime \prime}, c_{-1}^{\prime \prime \prime \prime}\right):=B\left(a_{1}^{\prime \prime \prime}, b_{0}^{\prime \prime \prime}, c_{-1}^{\prime \prime \prime}\right)$, then we have:

(1) $\quad\left(\tilde{p}_{10}+\tilde{p}_{11}\right) \bar{\partial} b_{0}^{\prime \prime \prime \prime}=\gamma^{-1} \psi\left(\tilde{p}_{10}+\tilde{p}_{11}\right) F \theta_{1}-\left(\tilde{p}_{10}+\tilde{p}_{11}\right)((\bar{\partial} \psi) \otimes F \theta)$;

(2) the assignment $b_{0}^{\prime \prime \prime} \mapsto \psi$ is $C^{\infty}(\bar{\Omega})$-linear.

COROLlary 3.6. We have the following.

(1) $d B A=d$

(2) If $b_{1}^{\prime} \in \mathcal{K}^{1,1}$, then $B A Q_{2}\left(a_{2}^{\prime}, b_{1}^{\prime}, c_{0}^{\prime}\right) \in \mathcal{K}^{0,1} \oplus \mathcal{K}^{1,0} \oplus K_{\bar{\Omega}}^{2,-1}$.

Proof. Part (1) is clear from the definitions of $A$ and $B$.

(2) Let $B A Q_{2}\left(a_{2}^{\prime}, b_{1}^{\prime}, c_{0}^{\prime}\right)=\left(a_{1}^{\prime \prime \prime \prime}, b_{0}^{\prime \prime \prime \prime}, c_{-1}^{\prime \prime \prime \prime}\right)$. Then, $b_{0}^{\prime \prime \prime \prime} \in \mathcal{K}^{1,0}$ follows from the definitions of $A, B$ and $Q_{2}$. Next, $b_{1}^{\prime}-q \circ \rho \circ R\left(b_{1}^{\prime}\right)=\bar{\partial} b_{0}^{\prime \prime \prime \prime}-F a_{1}^{\prime \prime \prime \prime}$ holds. Hence, if $\left(\tilde{p}_{10}+\tilde{p}_{11}\right) b_{1 \mid \partial \Omega}^{\prime}=0$, then $\left(\tilde{p}_{10}+\tilde{p}_{11}\right) F a_{1}^{\prime \prime \prime \prime}{ }_{\mid \partial \Omega}=0$ follows from Proposition 3.5(1). 
PROPOSITION 3.7. Any cohomology class of $H^{1}\left(K_{\bar{\Omega}}^{\bullet \bullet \bullet}\right)$ has a representative $\left(a_{1}, b_{0}, c_{-1}\right) \in \mathcal{K}^{0,1} \oplus \mathcal{K}^{1,0} \oplus K_{\bar{\Omega}}^{2,-1}$ having the following form:

$$
\begin{aligned}
& a_{1}=\phi_{0}+\psi \theta_{1}-\theta \otimes \bar{\partial} \psi, \\
& b_{0}=b_{0,0}-\psi F \theta
\end{aligned}
$$

for some $\phi_{0} \in A_{\bar{\Omega}}^{0,1}\left({ }^{\circ} \tilde{T}^{\prime} U\right), \psi \in C^{\infty}(\bar{\Omega})$ and $b_{0,0} \in A_{\bar{\Omega}}^{0}\left({ }^{\circ} \tilde{T}^{\prime} \mathbb{C}^{N} \mid U\right)$.

Proof. Let $\left(a_{1}, b_{0}, c_{-1}\right) \in K_{\bar{\Omega}}^{0,1} \oplus K_{\bar{\Omega}}^{1,0} \oplus K_{\bar{\Omega}}^{2,-1}$ satisfy $d\left(a_{1}, b_{0}, c_{-1}\right)=(0,0,0)$.

Denote $b_{0}=g \xi+Y$ where $g \in C^{\infty}(\bar{\Omega})$ and $Y \in A_{\bar{\Omega}}^{0}\left({ }^{\circ} \tilde{T}^{\prime} \mathbb{C}^{N}{ }_{\mid U}\right)$. Set $\tilde{a}_{0}:=(g / \gamma) \eta \in A_{\bar{\Omega}}^{0}\left(T^{\prime} U\right)$ and $\left(a_{1}^{(1)}, b_{0}^{(1)}, c_{-1}^{(1)}\right):=\left(a_{1}, b_{0}, c_{-1}\right)-d\left(\tilde{a}_{0}, 0,0\right)$ where we assumed $F(\eta)-\gamma \xi \in \Gamma\left(U,{ }^{\circ} \tilde{T}^{\prime} \mathbb{C}^{N}{ }_{\mid U}\right)$. Then $b_{0}^{(1)} \in A_{\bar{\Omega}}^{0}\left({ }^{\circ} \tilde{T}^{\prime} \mathbb{C}^{N} \mid U\right)$.

There exists $\tilde{a}_{0}^{(1)} \in A_{\bar{\Omega}}^{0}\left({ }^{\circ} \tilde{T}^{\prime} U\right)$ such that $\tilde{p}_{10} a_{1}^{(1)}=\tilde{p}_{10} \bar{\partial} \tilde{a}_{0}^{(1)}$ by (3.4). Let $\left(a_{1}^{(2)}, b_{0}^{(2)}, c_{-1}^{(2)}\right):=\left(a_{1}^{(1)}, b_{0}^{(1)}, c_{-1}^{(1)}\right)-d\left(\tilde{a}_{0}^{(1)}, 0,0\right)$. Then we have $\tilde{p}_{10} a_{1}^{(2)}=0$ and $b_{0}^{(2)} \in A_{\bar{\Omega}}^{0}\left({ }^{\circ} \tilde{T}^{\prime} \mathbb{C}^{N} \mid U\right)$.

Finally, let $\tilde{a}_{0}^{(2)}:=\left(\gamma \psi_{11}^{(2)}\right) \theta \in A_{\bar{\Omega}}^{0}\left(T^{1,0} U\right)$ and $\left(a_{1}^{(3)}, b_{0}^{(3)}, c_{-1}^{(3)}\right):=$ $\left(a_{1}^{(2)}, b_{0}^{(2)}, c_{-1}^{(2)}\right)-d\left(\tilde{a}_{0}^{(2)}, 0,0\right)$ where we denote $a_{1}^{(2)}=\psi_{0}^{(2)}+\psi_{11}^{(2)} \eta \otimes \bar{\eta}^{*}$ according to the decomposition (3.3). Then, we have $a_{1}^{(3)}=\psi_{0}^{(2)}-\bar{\partial}\left(\gamma \psi_{11}^{(2)}\right) \otimes \theta+\gamma \psi_{11}^{(2)}\left(\gamma^{-1} \eta \otimes\right.$ $\left.\bar{\eta}^{*}-\bar{\partial} \theta\right)$ and $b_{0}^{(3)}=b_{0}^{(2)}-\gamma \psi_{11}^{(2)} F \theta$. Hence, $a_{1}^{(3)} \in \mathcal{K}^{0,1}$ and $b_{0}^{(3)} \in \mathcal{K}^{1,0}$ follow from the property of $\theta$ as above, and it is clear that $a_{1}^{(3)}$ and $b_{0}^{(3)}$ have the desired decompositions.

In the final part of this section, we prove the main theorem.

THEOREM 3.8. There exist a family

$$
(\phi(t), f(t), k(t)) \in\left(\mathcal{K}^{0,1} \oplus \mathcal{K}^{1,0} \oplus K_{\bar{\Omega}}^{2,-1}\right)\left[\left[t_{1}, \ldots, t_{d}\right]\right]
$$

and an ideal $\mathfrak{I} \subset \mathbb{C}\left\{t_{1}, \ldots, t_{d}\right\}$ such that:

(1) $\quad(\phi(0), f(0), k(0))=(0,0,0)$;

(2) $\quad(\phi(t), f(t), k(t)) \equiv \sum_{\alpha=1}^{d}\left(\phi_{\alpha}, f_{\alpha}, k_{\alpha}\right) t_{\alpha} \bmod \mathfrak{m}^{2}$ where $\left\{\left[\left(\phi_{\alpha}, f_{\alpha}, k_{\alpha}\right)\right]\right\}_{\alpha=1}^{d}$ forms a cohomology basis of $H^{1}\left(K_{\bar{\Omega}}^{\bullet \bullet \bullet}\right)$ and $\mathfrak{m}$ denotes the maximal ideal of $\mathbb{C}\left\{t_{1}, \ldots, t_{d}\right\}$

(3) there exists an extension $\tilde{k}(t) \in H^{0}\left(\bar{B}(b), \mathcal{O}_{\mathbb{C}^{N}}\right)\left[\left[t_{1}, \ldots, t_{d}\right]\right]$ of $k(t)$ such that

$$
P(\phi(t), f(t), \tilde{k}(t)) \in \Im K_{\bar{\Omega}}^{2}\left[\left[t_{1}, \ldots, t_{d}\right]\right] ;
$$

(4) $\phi(t)$ is convergent with respect to $\|\cdot\|_{k}$-norm, $f(t)$ is convergent with respect to $\|\cdot\|_{k}$-norm and $\rho_{\mathcal{H}} P(\phi(t), f(t), \tilde{k}(t))$ is convergent with respect to the standard Euclidean norm; 
(5) $\mathfrak{I}$ is generated by $b_{1}(t), \ldots, b_{\ell}(t)$ where

$$
\rho_{\mathcal{H}} P(\phi(t), f(t), \tilde{k}(t))=\sum_{\beta=1}^{\ell} b_{\beta}(t) e_{\beta}
$$

for a fixed basis $e_{1}, \ldots, e_{\ell}$ of $\mathcal{H}^{2}$.

We prove Theorem 3.8 by modifying the formal construction in Section 2, using the adjustments in the first half of this section.

Let $\left\{\left(\phi_{\alpha}, f_{\alpha}, k_{\alpha}\right)\right\}_{1 \leq \alpha \leq d}$ be a system of cohomology basis of $H^{1}\left(K_{\bar{\Omega}}^{\bullet, \bullet}\right)$ having the property in Proposition 3.7, and set

$$
\left(\phi^{(1)}(t), f^{(1)}(t), k^{(1)}(t)\right)=\sum_{\alpha=1}^{d}\left(\phi_{\alpha}, f_{\alpha}, k_{\alpha}\right) t_{\alpha} .
$$

After determining $\left(\phi^{(\mu-1)}(t), f^{(\mu-1)}(t), k^{(\mu-1)}(t)\right)$ and $\tilde{k}^{(\mu-1)}(t)$, let $\left(\phi_{\mu}(t)\right.$, $\left.f_{\mu}(t), k_{\mu}(t)\right)$ denote the $\mu$ th homogeneous term of $-B \circ A \circ Q_{2} \circ Z_{2} \circ$ $P\left(\phi^{(\mu-1)}(t), f^{(\mu-1)}(t), \tilde{k}^{(\mu-1)}(t)\right)$ and denote

$$
\begin{aligned}
\left(\phi^{(\mu)}(t), f^{(\mu)}(t), k^{(\mu)}(t)\right):= & \left(\phi^{(\mu-1)}(t), f^{(\mu-1)}(t), k^{(\mu-1)}(t)\right) \\
& +\left(\phi_{\mu}(t), f_{\mu}(t), k_{\mu}(t)\right),
\end{aligned}
$$

where $Z_{2}$ and $Q_{2}$ (respectively $A$ and $B$ ) are the operators in Theorem 2.1 adjusted using the splitting (3.2) (respectively the operators in (3.5) and (3.6)).

Then, since $d B A=d$ holds, the argument of Section 2 works without any change and, hence, $(\phi(t), f(t), k(t)):=\lim _{\mu \rightarrow+\infty}\left(\phi^{(\mu)}(t), f^{(\mu)}(t), k^{(\mu)}(t)\right)$ and $\tilde{k}(t):=\lim _{\mu \rightarrow+\infty} \tilde{k}^{(\mu)}(t)$ provide a formal solution.

Next, we prove that $\phi(t), f(t)$ and $b_{1}(t), \ldots, b_{\ell}(t)$ are convergent. We prove it by the argument as in [K, Section 5.3(c)] and use the notation there. Let $\mathbf{K}$ be a Banach space with the norm $|\cdot| \mathbf{K}$. For $f(t)=\sum_{v_{1}, \ldots, v_{d}} f_{v_{1}, \ldots, v_{d}} t_{1}{ }^{v_{1}} \ldots t_{d} v_{d} \in$ $\mathbf{K}\left[\left[t_{1}, \ldots, t_{d}\right]\right]$, we define $|f|_{\mathbf{K}}(t)$ by $|f|_{\mathbf{K}}(t):=\sum_{v_{1}, \ldots, v_{d}}\left|f_{v_{1}, \ldots, v_{d}}\right|_{\mathbf{K}} t_{1}{ }^{v_{1}} \ldots t_{d}{ }^{v_{d}}$. For two $\mathbf{R}$-valued power series $a(t)=\sum_{v_{1}, \ldots, v_{d}} a_{v_{1}, \ldots, v_{d}} t_{1}{ }^{\nu_{1}} \ldots t_{d} v_{d}$ and $b(t)=$ $\sum_{v_{1}, \ldots, v_{d}} b_{v_{1}, \ldots, v_{d}} t_{1}^{v_{1}} \ldots t_{d} v_{d}$ with $a_{v} \geq 0, b_{v} \geq 0$, we write $a(t) \ll b(t)$ if $a_{v} \leq b_{v}$ holds for all $v_{1}, \ldots, v_{d}$. Let $A(t):=(b / 16 c) \sum_{\mu \geq 1}\left(c^{\mu} / \mu^{2}\right)\left(t_{1}+\cdots+t_{d}\right)^{\mu}$. Then $A(t)^{2} \ll(b / c) A(t)$ holds (cf. [K, (5.116)]).

We prove that

$$
\begin{gathered}
\|\phi\|_{k}(t) \ll A(t), \quad\|f\|_{k}(t) \ll A(t), \\
\left|b_{\gamma}\right|(t) \ll A(t) \quad(\gamma=1, \ldots, \ell)
\end{gathered}
$$

hold for some suitable $b>0$ and $c>0$. 
We denote by

$$
\begin{aligned}
\phi_{1}(t) & =\phi_{01}(t)+\psi_{1}(t) \theta_{1}-\theta \otimes \bar{\partial} \psi_{1}(t) \\
f_{1}(t) & =f_{01}(t)-\psi_{1}(t) F \theta
\end{aligned}
$$

the expressions of $\phi_{1}(t)$ and $f_{1}(t)$ in Proposition 3.7, and let $\chi_{1}(t)=\psi_{1}(t)=0$.

For $\mu \geq 2$, let $\left(a_{2 \mu}(t), b_{1 \mu}(t), c_{0 \mu}(t)\right)$ be the $\mu$ th homogeneous term of $-P\left(\phi^{(\mu-1)}(t), f^{(\mu-1)}(t), \tilde{k}^{(\mu-1)}(t)\right)$,

$$
\begin{aligned}
\chi_{\mu}(t) \otimes \xi & =\left(\tilde{p}_{10}+\tilde{p}_{11}\right) b_{1 \mu}(t), \\
\left(a_{2 \mu}^{\prime}(t), b_{1 \mu}^{\prime}(t), c_{0 \mu}^{\prime}(t)\right) & =Z_{2}\left(a_{2 \mu}(t), b_{1 \mu}(t), c_{0 \mu}(t)\right), \\
\left(a_{1 \mu}^{\prime \prime}(t), b_{0 \mu}^{\prime \prime}(t), c_{-1 \mu}^{\prime \prime}(t)\right) & =Q_{2}\left(a_{2 \mu}^{\prime}(t), b_{1 \mu}^{\prime}(t), c_{0 \mu}^{\prime}(t)\right), \\
\psi_{\mu}(t) \xi \otimes \bar{\eta}^{*} & =\tilde{p}_{11} \bar{\partial}\left(b_{0 \mu}^{\prime \prime}(t)-F \tilde{a}_{0 \mu}^{\prime \prime}(t)\right),
\end{aligned}
$$

where $\tilde{a}_{0 \mu}^{\prime \prime}(t)=L_{U}^{-1}\left(\tilde{p}_{10}\left(\bar{\partial} b_{0 \mu}^{\prime \prime}(t)\right)\right)$. Then, we have the following proposition.

Proposition 3.9. We have the following.

(1) There exist $\phi_{0 \mu}(t) \in A_{\bar{\Omega}}^{0,1}\left({ }^{\circ} \tilde{T}^{\prime} U\right)[t]$ and $f_{0 \mu} \in A_{\bar{\Omega}}^{0}\left({ }^{\circ} \tilde{T}^{\prime} \mathbb{C}^{N}{ }_{\mid U}\right)[t]$ such that the following equations hold:

$$
\begin{aligned}
\phi_{\mu}(t) & =\phi_{0 \mu}(t)-(1 / \gamma) \chi_{\mu}(t) \otimes \eta-\psi_{\mu}(t) \theta_{1}-\theta \otimes \bar{\partial} \psi_{\mu}(t), \\
f_{\mu}(t) & =f_{0 \mu}(t)-\psi_{\mu}(t) F \theta .
\end{aligned}
$$

(2) For a fixed neighbourhood $U^{\prime}$ on which ${ }^{\circ} \tilde{T}^{\prime} U$ is trivialized, there exist $\chi_{\mu I}(t), \quad \chi_{\mu I I I}(t) \in A_{\bar{\Omega}}^{0,1}[t], \quad \chi_{\mu I I, \lambda}(t) \in A_{U^{\prime} \cap \bar{\Omega}}^{0,1}[t](1 \leq \lambda \leq n-1)$ and $\chi_{\mu I V}(t) \in C^{\infty}(\bar{\Omega})[t]$ such that

$$
\begin{aligned}
\chi_{\mu}(t)= & \chi_{\mu I}(t) F \theta(r)+\sum_{\lambda=1}^{n-1} \chi_{\mu I I, \lambda}(t) e_{\lambda}(F \theta(r))+\chi_{\mu I I I}(t) \theta(F \theta(r)) \\
& +\chi_{\mu I V}(t) \theta_{1}(F \theta(r))
\end{aligned}
$$

holds on $U^{\prime} \cap \bar{\Omega}$ with a local frame $\left\{e_{\lambda}\right\}_{1 \leq \lambda \leq n-1}$ of ${ }^{\circ} \tilde{T}^{\prime} U$ over $U^{\prime}$, and that

- $r \chi_{\mu I}(t)$ the $\mu$ th homogeneous term of

$$
\begin{aligned}
& \phi_{0}^{(\mu-1)}(t)\left(\psi^{(\mu-1)}(t)\right)+\psi^{(\mu-1)}(t) \theta_{1}\left(\psi^{(\mu-1)}(t)\right) \\
& +\left(\bar{\partial} \psi^{(\mu-1)}(t)\right) \theta\left(\psi^{(\mu-1)}(t)\right)-(1 / \gamma) \chi^{(\mu-1)}(t) \eta\left(\psi^{(\mu-1)}(t)\right) \\
& -(1 / \gamma) \chi_{I}^{(\mu-1)}(t) \psi^{(\mu-1)}(t) \eta(F \theta(r)) ;
\end{aligned}
$$


- $r \chi_{\mu I I, \lambda}(t)$ is the $\mu$ th homogeneous term of

$$
\psi^{(\mu-1)}(t) \phi_{0}^{(\mu-1) \lambda}(t)-(1 / \gamma) \chi_{I I, \lambda}^{(\mu-1)}(t) \psi^{(\mu-1)}(t) \eta(F \theta(r)) ;
$$

- $r \chi_{\mu I I I}(t)$ is the $\mu$ th homogeneous term of

$$
\psi^{(\mu-1)}(t)\left(\bar{\partial} \psi^{(\mu-1)}(t)\right)-(1 / \gamma) \chi_{I I I}^{(\mu-1)}(t) \psi^{(\mu-1)}(t) \eta(F \theta(r)) ;
$$

- $r \chi_{\mu I V}(t)$ is the $\mu$ th homogeneous term of

$$
\psi^{(\mu-1)}(t) \psi^{(\mu-1)}(t)-(1 / \gamma) \chi_{I V}^{(\mu-1)}(t) \psi^{(\mu-1)}(t) \eta(F \theta(r))
$$

hold, where we denote $\phi_{0}^{(\mu-1)}(t)=\sum_{\nu=1}^{\mu-1} \phi_{\nu 0}(t)$ and use the same notation for $\chi_{I}^{(\mu-1)}(t), \chi_{I I, \lambda}^{(\mu-1)}(t), \chi_{I I I}^{(\mu-1)}(t), \chi_{I V}^{(\mu-1)}(t)$ and $\psi^{(\mu-1)}(t)$, and $\left\{\phi_{0}^{(\mu-1) \lambda}(t)\right\}_{1 \leq \lambda \leq n-1}$ are given by $\phi_{0}^{(\mu-1)}(t)=\sum_{\lambda=1}^{n-1} \phi_{0}^{(\mu-1) \lambda}(t) e_{\lambda}$.

Proof. (1) First, we note that

$$
\begin{aligned}
f_{\mu}(t) & =b_{0 \mu}^{\prime \prime}(t)-F \tilde{a}_{0 \mu}^{\prime \prime}(t)-\psi_{\mu}(t) F \theta, \\
F \phi_{\mu}(t) & =\bar{\partial} f_{\mu}(t)-b_{1 \mu}^{\prime}(t) .
\end{aligned}
$$

Since $b_{0 \mu}^{\prime \prime}(t) \in A_{\bar{\Omega}}^{0}\left({ }^{\circ} \tilde{T}^{\prime} \mathbb{C}^{N} \mid U\right)[t]$ and $\tilde{a}_{0 \mu}^{\prime \prime}(t) \in A_{\bar{\Omega}}^{0}\left({ }^{\circ} \tilde{T}^{\prime} U\right)[t]$, we have $f_{0 \mu}(t):=$ $f_{\mu}(t)+\psi_{\mu}(t) F \theta=b_{0 \mu}^{\prime \prime}(t)-F \tilde{a}_{0 \mu}^{\prime \prime}(t) \in A_{\bar{\Omega}}^{0}\left({ }^{\circ} \tilde{T}^{\prime} \mathbb{C}^{N} \mid U\right)[t]$.

Next, since

$$
\begin{aligned}
F \phi_{\mu}(t)(r) & =\psi_{\mu}(t) r \bar{\eta}^{*}-\bar{\partial}\left(\psi_{\mu}(t) F \theta\right)(r)-\chi_{\mu}(t) r \\
& =F\left(\psi_{\mu}(t)\left(\frac{1}{\gamma} \eta \otimes \bar{\eta}^{*}-\bar{\partial} \theta\right)-\bar{\partial} \psi_{\mu}(t) \otimes \theta-\chi_{\mu}(t) \otimes\left(\frac{\eta}{\gamma}\right)\right)(r),
\end{aligned}
$$

we have

$$
\phi_{\mu}(t)-\psi_{\mu}(t) \theta_{1}-\bar{\partial} \psi_{\mu}(t) \otimes \theta-(1 / \gamma) \chi_{\mu}(t) \otimes \eta \in A_{\bar{\Omega}}^{0}\left({ }^{\circ} \tilde{T}^{\prime} U\right)[t] .
$$

(2) We prove the assertion by induction on $\mu$. Since $\chi_{1}(t)=0$, it is enough to prove it under the existence of $\chi_{\nu I}(t), \chi_{\nu I I, \lambda}(t), \chi_{\nu I I I}(t), \chi_{v I V}(t)$ for all $1 \leq v \leq$ $\mu-1$. Note that $b_{1 \mu}(t)(r)=r \chi_{\mu}(t)$ because $\xi(r)=r$ holds. Since $b_{1 \mu}(t)$ is the $\mu$ th homogeneous term of $-\phi^{(\mu-1)}(t) f^{(\mu-1)}(t)$ and since $\phi\left(f_{0}\right)(r)=0$ holds for $\phi \in A_{\bar{\Omega}}^{0,1}\left(T^{1,0} U\right)$ and $f_{0} \in A_{\bar{\Omega}}^{0}\left({ }^{\circ} \tilde{T}^{\prime} \mathbb{C}^{N} \mid U\right)$ by $(1)$, we have that $b_{1 \mu}(t)(r)$ is the $\mu$ th 
homogeneous term of

$$
\begin{aligned}
& \left\{\phi_{0}^{(\mu-1)}(t)\left(\psi^{(\mu-1)}(t)\right)+\psi^{(\mu-1)}(t) \theta_{1}\left(\psi^{(\mu-1)}(t)\right)+\left(\bar{\partial} \psi^{(\mu-1)}(t)\right) \theta\left(\psi^{(\mu-1)}(t)\right)\right\} F \theta(r) \\
& +\psi^{(\mu-1)}(t) \phi_{0}^{(\mu-1)}(t)(F \theta(r))+\psi^{(\mu-1)}(t) \psi^{(\mu-1)}(t) \theta_{1}(F \theta(r)) \\
& +\psi^{(\mu-1)}(t) \bar{\partial} \psi^{(\mu-1)}(t) \theta(F \theta(r))-(1 / \gamma) \chi^{(\mu-1)}(t) \eta\left(\psi^{(\mu-1)}(t)\right) F \theta(r) \\
& -(1 / \gamma) \chi^{(\mu-1)}(t) \psi^{(\mu-1)}(t) \eta(F \theta(r)) .
\end{aligned}
$$

Then, by the inductive assumption, we have the assertion.

Now we prove the convergence of $\phi(t), f(t)$ and $b_{\gamma}(t)$. We fix a finite open covering $\bar{\Omega} \subset \bigcup_{i \in \Lambda} U_{i}$ such that ${ }^{\circ} \tilde{T}^{\prime} U$ is differentiably trivialized on $U_{i}$. Let $\|\phi\|_{k}$ denote the Sobolev $k$-norm of $\phi \in A_{\bar{\Omega}}^{0,1}\left(T^{\prime} U\right)$ and introduce the norms $\|\chi\|_{\{k\}}$ for $\chi \in A_{\bar{\Omega}}^{0,1},\|\psi\|_{k}^{\prime}$ for $\psi \in C^{\infty}(\bar{\Omega})$ and $\|f\|_{k}^{\prime}$ for $f \in A_{\bar{\Omega}}^{0}\left(T^{\prime} \mathbb{C}_{\mid U}^{N}\right)$ as follows: on a neighbourhood $U_{i}$, we fix a local frame $\left\{e_{\lambda}\right\}_{1 \leq \lambda \leq n-1}$ of ${ }^{\circ} \tilde{T}^{\prime} U$. For $\chi \in A_{\bar{\Omega}}^{0,1}$ with Supp $\chi \subset U_{i}$,

$$
\|\chi\|_{\{k\}}:=\left\|\chi_{I}\right\|_{k}+\sum_{\lambda=1}^{n-1}\left\|\chi_{I I, \lambda}\right\|_{k}+\left\|\chi_{I I I}\right\|_{k}+\left\|\chi_{I V}\right\|_{k} .
$$

For $\psi \in A_{\bar{\Omega}}^{0,1}$ with $\operatorname{Supp} \psi \subset U_{i}$,

$$
\begin{aligned}
\|\psi\|_{k}^{\prime}:= & \|\bar{\partial} \psi\|_{k}+\sum_{\lambda=1}^{n-1}\left\|e_{\lambda}(\psi)\right\|_{k}+\|\theta \psi\|_{k}+\left\|\theta_{1} \psi\right\|_{k} \\
& +\|(F(\theta(r))) \eta \psi\|_{k}+\sum_{\lambda=1}^{n-1}\left\|\left(e_{\lambda}(F(\theta(r)))\right) \eta \psi\right\|_{k} \\
& +\|(\theta(F \theta(r))) \eta \psi\|_{k}+\left\|\left(\theta_{1}(F \theta(r))\right) \eta \psi\right\|_{k}+\|\psi\|_{k} .
\end{aligned}
$$

$\|f\|_{k}^{\prime}$ is defined similarly for $f \in A_{\bar{\Omega}}^{0}\left(T^{\prime} \mathbb{C}_{\mid U}^{N}\right)$ with Supp $f \subset U_{i}$.

For global $\chi, \psi$ and $f,\|\chi\|_{\{k\}},\|\psi\|_{k}^{\prime}$ and $\|f\|_{k}^{\prime}$ are defined by patching the local definitions using a partition unity. Here, we note that $(F \theta(r))_{\mid \partial \Omega}=\left(e_{\lambda} F \theta(r)\right)_{\mid \partial \Omega}=$ $(\theta F \theta(r))_{\mid \partial \Omega}=0$ and $\left(\theta_{1} F \theta(r)\right)_{\mid \partial \Omega}=0$ hold.

We shall prove

$$
\begin{aligned}
\left\|\phi^{(\mu)}\right\|_{k}(t) & \ll A(t), \\
\left\|\chi^{(\mu)}\right\|_{\{k\}}(t) & \ll A(t), \\
\left\|f^{(\mu)}\right\|_{k}^{\prime}(t) & \ll A(t), \\
\left\|\psi^{(\mu)}\right\|_{k}^{\prime}(t) & \ll A(t)
\end{aligned}
$$

for $\mu=1,2, \ldots$. 
(3.9) ${ }_{1}$ holds if we choose $b$ sufficiently large. Suppose that $(3.9)_{\mu-1}(\mu \geq 2)$ holds for some $b>0, c>0$.

Proposition 3.10. There exist positive constants $C_{1}, \ldots, C_{11}$ independent of $\mu$ and such that:

(1) $\left\|b_{1 \mu}\right\|_{k}(t) \ll \frac{b}{c} C_{1} A(t)$;

(2) $\left\|b_{1 \mu}^{\prime}\right\|_{k}(t) \ll \frac{b}{c} C_{2} A(t)$;

(3) $\left\|b_{0 \mu}^{\prime \prime}\right\|_{k}^{\prime}(t) \ll \frac{b}{c} C_{3} A(t)$;

(4) $\left\|a_{1 \mu}^{\prime \prime}\right\|_{k}(t) \ll \frac{b}{c} C_{4} A(t)$;

(5) $\left\|\bar{\partial} a_{0 \mu}^{\prime \prime}\right\|_{k}(t) \ll \frac{b}{c} C_{5} A(t)$;

(6) $\left\|\chi_{\mu}\right\|_{\{k\}}(t) \ll \frac{b}{c} C_{6} A(t)$;

(7) $\left\|\psi_{\mu}\right\|_{k}^{\prime}(t) \ll \frac{b}{c} C_{7} A(t)$;

(8) $\left\|f_{0 \mu}\right\|_{k}^{\prime}(t) \ll \frac{b}{c} C_{8} A(t)$;

(9) $\left\|f_{\mu}\right\|_{k}^{\prime}(t) \ll \frac{b}{c} C_{9} A(t)$;

(10) $\left\|\phi_{\mu}\right\|_{k}(t) \ll \frac{b}{c} C_{10} A(t)$;

(11) $\left\|\phi_{0 \mu}\right\|_{k}(t) \ll \frac{b}{c} C_{11} A(t)$.

Proof. (1) Since

$$
\begin{aligned}
b_{1 \mu}(t) & \\
= & \left(\phi_{0}^{(\mu-1)}(t)-\frac{1}{\gamma} \chi^{(\mu-1)}(t) \otimes \eta-\psi^{(\mu-1)}(t) \theta_{1}-\theta \otimes \bar{\partial} \psi^{(\mu-1)}(t)\right) \\
& \cdot\left(f_{0}^{(\mu-1)}(t)-\psi^{(\mu-1)}(t) F \theta\right) \\
= & \phi_{0}^{(\mu-1)}(t)\left(f_{0}^{(\mu-1)}(t)\right)-\phi_{0}^{(\mu-1)}(t)\left(\psi^{(\mu-1)}(t)\right) F \theta-\psi^{(\mu-1)}(t) \phi_{0}^{(\mu-1)}(t)(F \theta) \\
& -\frac{1}{\gamma} \chi^{(\mu-1)}(t) \eta\left(f_{0}^{(\mu-1)}(t)\right)+\frac{1}{\gamma} \chi^{(\mu-1)}(t) \eta\left(\psi^{(\mu-1)}(t)\right) F \theta \\
& +\frac{1}{\gamma} \chi^{(\mu-1)}(t) \psi^{(\mu-1)}(t) \eta(F \theta) \\
& -\psi^{(\mu-1)}(t) \theta_{1}\left(f_{0}^{(\mu-1)}(t)\right)+\psi \psi^{(\mu-1)}(t) \theta_{1}\left(\psi^{(\mu-1)}(t)\right) F \theta \\
& +\psi^{(\mu-1)}(t) \psi^{(\mu-1)}(t) \theta_{1}(F \theta) \\
& -\left(\bar{\partial} \psi^{(\mu-1)}(t)\right) \theta\left(f_{0}^{(\mu-1)}(t)\right)+\left(\bar{\partial} \psi^{(\mu-1)}(t)\right) \theta\left(\psi^{(\mu-1)}(t)\right) F \theta \\
& +\left(\bar{\partial} \psi^{(\mu-1)}(t)\right) \psi^{(\mu-1)}(t) \theta(F \theta),
\end{aligned}
$$


we have

$$
\begin{aligned}
& \left\|b_{1 \mu}\right\|_{k}(t) \\
& \ll C\left(\left\|\phi_{0}^{(\mu-1)}\right\|_{k}(t)\left\|f_{0}^{(\mu-1)}\right\|_{k}^{\prime}(t)+\left\|\phi_{0}^{(\mu-1)}\right\|_{k}(t)\left\|\psi^{(\mu-1)}\right\|_{k}^{\prime}(t)\right. \\
& \left.\quad+\left\|\psi^{(\mu-1)}\right\|_{k}^{\prime}(t)\left\|f_{0}^{(\mu-1)}\right\|_{k}^{\prime}(t)+\left\|\chi^{(\mu-1)}\right\|_{k}(t)\left\|\psi^{(\mu-1)}\right\|_{k}(t)+\left\|\psi^{(\mu-1)}\right\|_{k}^{\prime 2}(t)\right) \\
& \quad+C^{\prime}\left(\left\|\left(\chi^{(\mu-1)}(t) \eta\right) f_{0}^{(\mu-1)}(t)\right\|_{k}+\left\|\left(\chi^{(\mu-1)}(t) \eta\right) \psi^{(\mu-1)}(t)\right\|_{k}\right)
\end{aligned}
$$

for some positive constants $C$ and $C^{\prime}$ independent of $\mu$. By Proposition 3.9(2) and inductive assumption, we have

$$
\left\|\left(\chi^{(\mu-1)}(t) \eta\right) f_{0}^{(\mu-1)}(t)\right\|_{k},\left\|\left(\chi^{(\mu-1)}(t) \eta\right) \psi^{(\mu-1)}(t)\right\|_{k} \ll C^{\prime \prime} A(t)^{3}
$$

for some positive constant $C^{\prime \prime}$ independent of $\mu$. Hence, we have

$$
\left\|b_{1 \mu}\right\|_{k}(t) \ll C^{\prime \prime \prime} A(t)^{2}+C^{\prime \prime \prime \prime} A(t)^{3} \ll\left(\frac{b}{c} C^{\prime \prime \prime}+\left(\frac{b}{c}\right)^{2} C^{\prime \prime \prime \prime}\right) A(t) .
$$

Parts (2)-(4) are clear from (1) and Proposition 2.4. Part (5) follows from (3) and Remark 3.3. Part (6) follows from Proposition 3.9(2) and the inductive assumption. Part (7) follows from (3) and Proposition 3.5(2) and Remark 3.3. Part (8) follows from (3) and Remark 3.3 since $f_{0 \mu}(t)=b_{0 \mu}^{\prime \prime}(t)-F \tilde{a}_{0 \mu}^{\prime \prime}(t)$. Part (9) follows from (7) and (8). Part (10) follows from (2) and (9) since $F \phi_{\mu}(t)=\bar{\partial} f_{\mu}(t)-b_{1 \mu}^{\prime}(t)$. Part (11) follows from (6), (7) and (10) using the equation in Proposition 3.9.

Therefore, if $c>2 b \max \left\{C_{1}, \ldots, C_{11}\right\}$, then (3.9) $\mu$ holds for all $\mu \geq 1$. By the same technique as in the CR-case (cf. [Mi3, Section 8]), we have

$$
\left|b_{\gamma}\right|(t) \ll A(t) \quad(\gamma=1, \ldots, \ell) .
$$

Therefore we obtained $\phi(t), f(t), \tilde{k}(t)$ and $b_{1}(t), \ldots, b_{\ell}(t)$ satisfying (1)-(5) of Theorem 3.8.

By the same technique as in [Mi3, Section 10], we can prove the following Kuranishi-versality.

PROPOSITION 3.11. Let $(\phi(t), f(t))$ be the stably embeddable family of deformations of the complex structure on $\bar{\Omega}$ constructed in Theorem 3.8. Let $\pi^{\prime}: \mathcal{X}^{\prime} \rightarrow T^{\prime}$ be a family of complex manifolds with an embedding

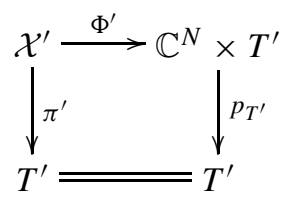


satisfying $p_{T^{\prime}} \circ \Phi^{\prime}=\pi^{\prime}$ and such that $\mathcal{X}_{*}^{\prime}$ is a neighbourhood of $\bar{\Omega}$ in $U$ and $\Phi_{\mid \mathcal{X}_{*}^{\prime}}^{\prime}=$ $f_{0 \mid \mathcal{X}_{*}^{\prime}}$ for some $* \in T$. Then there exists a holomorphic map $\tau:\left(T^{\prime}, *\right) \rightarrow(T, 0)$, a holomorphic family of embeddings $G: \bar{\Omega} \times T^{\prime} \rightarrow \mathcal{X}^{\prime}$ and a formal family of holomorphic maps $\hat{\eta}: \bar{B} \times T^{\prime} \rightarrow \mathbb{C}^{N} \times T^{\prime}$ satisfying:

(1) $\quad \tau(*)=0, G_{\mid \bar{\Omega} \times *}=i d_{\bar{\Omega}}$ and $\hat{\eta}_{\mid B \times *}=i d_{\mathbb{C}^{N} \mid \bar{B}}$;

(2) the family of complex structures $\phi\left(\tau\left(t^{\prime}\right)\right)$ is induced by the holomorphic family of embeddings $G$;

(3) $\pi^{\prime} \circ G=p_{T^{\prime}}$ and the formal family of embeddings $\hat{\eta} \circ \Phi^{\prime} \circ G: \bar{\Omega} \times T^{\prime} \rightarrow \mathbb{C}^{N} \times T^{\prime}$ coincides with $f_{0}+f\left(\tau\left(t^{\prime}\right)\right)$.

The proof of Main Theorem will be completed by showing the following proposition.

Proposition 3.12. Let $\varpi: \mathcal{V} \rightarrow S$ be the versal family of flat deformation of $V$. Then $(T, 0) \simeq(S, 0)$ holds and there exists a holomorphic family of embeddings $G: \bar{\Omega} \times T \rightarrow \mathcal{V}$ such that:

(1) $G_{\mid \bar{\Omega} \times 0}: \bar{\Omega} \rightarrow \mathcal{V}_{0}$ coincides with $f_{0 \mid \bar{\Omega}}: \bar{\Omega} \rightarrow \mathbb{C}^{N}$;

(2) $\phi(t)$ is induced from the embedding $G: \bar{\Omega} \times T \rightarrow \mathcal{V}$.

We omit the proofs of these propositions since they are parallel to the arguments of Sections 10 and 11 of [Mi3], respectively.

Acknowledgements. Research partially supported by Grant-in-Aid for Scientific Research (No. 14540087).

\section{REFERENCES}

[Ad] K. Adachi. Continuation of holomorphic functions from subvarieties to pseudoconvex domains. Kobe J. Math. 11 (1994), 33-47.

[Ak1] T. Akahori. The new Neumann operator associated with deformations of strongly pseudo convex domains and its application to deformation theory. Invent. Math. 68 (1982), 317-352.

[Ak2] T. Akahori. A criterion for the Neumann type problem over a differential complex on a strongly pseudo convex domain. Math. Ann. 264 (1983), 525-535.

[BGS] R. Beals, P. C. Greiner and N. K. Stanton. $L^{p}$ and Lipschitz estimates for the $\bar{\partial}$-equation and the $\bar{\partial}$-Neumann problem. Math. Ann. 277 (1987), 185-196.

[BE] J. Bland and C. L. Epstein. Embeddable CR-structures and deformations of pseudoconvex surfaces Part I: Formal deformations. J. Alg. Geom. 5 (1996), 277-368.

[GS] P. C. Greiner and E. M. Stein. Estimates for the $\bar{\partial}$-Neumann Problem (Mathematical Notes, 19). Princeton University Press, Princeton, NJ, 1977. 
[Ho] L. Hörmander. $L^{2}$-estimates and existence theorems for the $\bar{\partial}$-operator. Acta Math. J. 113 (1965), 89-152.

[K] K. Kodaira. Complex Manifolds and Deformation of Complex Structures (Die Grundlehren der Mathematischen Wissenshaften, 283). Springer, Berlin, 1981.

[Mi1] K. Miyajima. Deformations of a complex manifold near a strongly pseudo-convex real hypersurface and a realization of Kuranishi family of storngly pseudo-convex CR structures. Math. Z. 205 (1990), 593-602.

[Mi2] K. Miyajima. Deformations of strongly pseudo-convex CR structures and deformations of normal isolated singularities. Complex Analysis (Aspects of Mathematics, E16). Ed. K. Diederich. Vieweg, Braunschweig, 1992, pp. 200-204.

[Mi3] K. Miyajima. CR construction of the flat deformations of normal isolated singularities. J. Alg. Geom. 8 (1999), 403-470.

Kimio Miyajima

Department of Mathematics and Computer Science

Faculty of Science

Kagoshima University

Kagoshima 890-0065

Japan

(E-mail:miyajima@sci.kagoshima-u.ac.jp) 